\title{
Isogeometric boundary element methods for three dimensional static fracture and fatigue crack growth
}

\author{
X. Peng ${ }^{\text {a }}$, E. Atroshchenko ${ }^{\text {b,* }}$, P. Kerfriden ${ }^{\text {a }}$, S.P.A. Bordas ${ }^{\text {c,d,a,e }}$ \\ ${ }^{a}$ Institute of Mechanics Materials and Advanced Manufacturing, Cardiff University, CF24 3AA, UK \\ ${ }^{\mathrm{b}}$ Department of Mechanical Engineering, University of Chile, Santiago, 8370448, Chile \\ ${ }^{\mathrm{c}}$ Université du Luxembourg, Faculté des Sciences, de la Technologie et de la Communication, 6 rue Richard Coudenhove-Kalergi, L-1359, \\ Luxembourg \\ ${ }^{\mathrm{d}}$ Research Unit in Engineering Science Campus Kirchberg, G 007, Luxembourg \\ e University of Western Australia, 35 Stirling Hwy, Crawley, WA 6009, Australia
}

\section{Highlights}

- We implement an isogeometric BEM routine for fracture by use of dual boundary integral equations.

- We propose a singular integration scheme to improve the quadrature accuracy for elements with high aspect ratios.

- We investigate the approaches to compute stress intensity factors based on a NURBS representation of the crack surfaces.

- We outline a geometric algorithm to propagate the crack based on the fatigue Paris law.

\begin{abstract}
We present a novel numerical method to simulate crack growth in 3D, directly from the Computer-Aided Design (CAD) geometry of the component, without any mesh generation. The method is an isogeometric boundary element method (IGABEM) based on non-uniform rational B-splines (NURBS). NURBS basis functions are used for the domain and crack representation as well as to approximate the physical quantities involved in the simulations. A stable quadrature scheme for singular integration is proposed to enhance the robustness of the method in dealing with highly distorted elements. Convergence studies in the crack opening displacement is performed for a penny-shaped crack and an elliptical crack. Two approaches to extract stress intensity factors (SIFs): the contour $M$ integral and the virtual crack closure integral are compared using dual integral equations. The results show remarkable accuracy in the computed SIFs, leading to smooth crack paths and reliable fatigue lives, without requiring the generation of any mesh from the CAD model of the component under consideration.
\end{abstract}

(c) 2016 Elsevier B.V. All rights reserved.

Keywords: Isogeometric analysis; NURBS; Linear elastic fracture; Boundary element method; Crack growth; Fracture simulations directly from CAD

\footnotetext{
* Corresponding author. Fax: +56 26896057.

E-mail address: eatroshch@gmail.com (E. Atroshchenko).
} 


\section{Introduction}

\subsection{Damage tolerance assessment}

The simulation of crack propagation is crucial in most areas of engineering and science. Predicting fracture is central to devising more durable and safer products and to understand natural phenomena.

We focus in this paper on one particular type of failure simulation known as 'fatigue crack growth' and which occurs in a variety of fields. When subjected to low amplitude cyclic loading, much below those causing significant unrecoverable plastic deformations, materials gradually damage which leads to crack initiation, propagation, and, in turn, to the subsequent global failure of the component.

In fatigue crack growth simulations, the goal is to estimate the number of cycles that a component can undergo before it is unfit for purpose. Evaluating this 'residual life' requires the ability, to both compute the number of cycles to initiation and to estimate the growth of a given crack given a number of loading cycles. We do not discuss the crack initiation phase in this paper, but focus on the problem of simulating the growth of a crack whose initial geometry is known. One of the widely used approaches to predict fatigue crack propagation consists in assuming that the material around the crack behaves in a linear elastic manner, compute the energy release rate, through the evaluation of stress intensity factors, and based on those, to estimate the amount of crack advance using empirical laws such as the Paris law.

\subsection{Simulating fatigue crack growth}

Simulating (fatigue) crack growth leads to a number of requirements for the associated numerical methods ${ }^{1}$ :

1. Computing the displacement and stress fields within the component during crack growth;

2. Representing the crack surface as it grows;

3. Estimating the energy release rate along the crack;

4. Computing the crack propagation angle and crack propagation increment along the crack front.

Point 1 . is complicated by the singularity of the stress field along the crack front, which requires dense meshes to be generated and regenerated as the crack grows. Point 2. requires the numerical method to deal with the propagating displacement discontinuity across the crack faces, as those evolve. Point 3. assumes the ability of the method to accurately evaluate stress intensity factors (SIFs). In this paper, we consider two approaches to do so, which are detailed later in the manuscript. The crack growth direction and increment (Point 4.) is provided by the crack growth law. We assume in the following that the crack grows in the direction which maximizes the tangential stress (hoop stress). We use Paris' law to compute the increment in crack growth along the crack front. We focus in the rest of this introduction on points 1. and 2. only, for which a number of methods have been proposed over the years. Points 1 . and 2. above represent the major challenge for numerical fracture modeling, i.e. the combined requirement of simulating evolving discontinuities and singularities. This requires meshes used in the finite element method to be generated and regenerated as the crack grows and to use special elements relying, e.g. on Barsoum elements [2] to represent the singularity. This difficulty is compounded by the fact that the cracks are typically two or more orders of magnitude smaller than the component itself which exacerbates the meshing difficulty by leading to large gradients in element size.

In spite of these challenges, the finite element method (FEM) has been used rather widely to simulate the crack propagation directly with certain adaptive re-meshing operation [3-5]. Some commercial software packages such as ADAPCRACK, ZENCRACK and FRANC3D have been developed based on this idea [6-8] and two recent review papers can be consulted for details [1,9]. The complexity of remeshing, in spite of tremendous recent progress in Delaunay triangulation [10] and other meshing procedures [11], re-meshing remains a difficult problem when multiple cracks are interacting or for geometrically complex components where the presence of cracks as internal boundaries significantly constrain the mesh generation and regeneration process.

\footnotetext{
${ }^{1}$ Note that because realistic industrial problems are three dimensional, we will not consider methods which have been developed only for two-dimensional crack propagation. We refer the reader to a recent review on 3D crack growth for some details which are not included in the forthcoming literature review [1].
} 
Partition of unity enrichment volume-based methods. Another approach is to introduce cracks through enrichment functions using the partition of unity method [12]. This was first done in a method called the 'extended finite element method' [13] and the 'generalized finite element method' [14,15] which are both relying on the idea of multiplying the standard shape functions by enrichment functions which 'know' about the nature of the solution. Whilst enrichment itself is an idea dating back to the 1970's [16], and is also found in the meshfree literature since the early 1990's [17] ${ }^{2}$ partition of unity enrichment enables a local enrichment of the basis, leading to banded matrices and enabling to flexibly combine different enrichments within a single computational domain. This final point is particularly important as enrichment locality is required to enable the simulation of multiple crack fronts.

In practice, partition of unity (PU) enrichment is an effective approach to somewhat alleviate the meshing and remeshing burden in fracture modeling [13-15]. Additional enrichment functions are used to both introduce displacement discontinuities and stress singularities into the model. This enables to introduce the cracks quasiindependently of the background mesh. This flexibility simplifies local adaptive processes, as the regenerated mesh need not conform to the crack faces [19-21] does not come for free, as (1) numerical integration requires special attention [22-24]; (2) optimal convergence requires enriching a volume independent of the mesh size, thereby deteriorating the condition number [25-27]; (3) since the mesh does not conform to the crack, the latter must be represented geometrically through another approach. Usually, enrichment is coupled with level set methods [28] used to represent the crack surfaces implicitly. This has been used for 3D crack growth problems [29-31,27] as well as for industrial applications [32,21,33]. A special case of partition of unity methods, meshfree methods have also been proposed with the aim of further reducing the mesh burden, for example, the element-free Galerkin (EFG) [34], the extended EFG [35,36], the cracking-particle method [37] etc. For more details, the readers could refer the review paper on meshfree methods and their implementation, by Nguyen et al. [18] and the review paper on partition of unity methods for fracture in [1].

Boundary-based methods. Another approach is to model cracks by boundary-based methods such as the boundary element method (BEM) or the scaled boundary finite element method (SBFEM).

Boundary element methods can be collocation or Galerkin-based. For problems without body forces or internal variables, the discretization of the boundary is sufficient to approximate the field variables. When cracks evolve, only the boundary surfaces are updated which does not require the regeneration of a volume mesh. Several additional difficulties come with the flexibility brought by boundary-based methods.

1. The fundamental solution to the problem at hand must be known, which limits the applicability to the method rather severely. However, for many industrial damage tolerance problems, linear elastic fracture is used, which makes boundary-based methods generally applicable.

2. Particular care is required to deal with the fact that the cracks are zero-thickness surfaces, which leads to overlapping collocation points and thus identical equations. To avoid the resulting singularity in the system matrix, Hong and Chen [38] proposed the dual boundary integral representations by introducing the hyper-singular equation derived from the secondary field [39].

3. Numerical integration is complicated, due to the emergence of hyper-singular terms in the integrals. However, a number of methods have been proposed to deal with these singularities. The most common ones are singularity subtraction techniques (used in this paper) and regularization of the boundary integral equations[40,41].

4. The resulting system matrices are full. This makes the solution of the system of equations slow. However, this is not specific to fracture mechanics and approaches have been proposed to accelerate the solution process [42].

The use of dual boundary integral equations makes the crack propagation simulation more effective through a single domain. And the corresponding dual BEM was subsequently implemented for 2D and 3D fracture [43-45] and was extended to material-nonlinear fracture [46,47] and dynamic crack propagation [48]. Commercial packages based on BEM are BEASY [49] and FRANC3D [8]. Apart from the dual BEM based on the collocation method, the Galerkin BEM, in particular the symmetric Galerkin BEM (SGBEM) has also drawn attention for fracture analysis [50-52]. The symmetric matrix system of SGBEM also facilitates the coupling with FEM [53,54].

An interesting hybrid approach was proposed named scaled boundary finite element method (SBFEM) by Song and Wolf [55]. Only the boundary of the domain is needed for discretization in analysis without fundamental solution. Thus

\footnotetext{
2 [18] provides a review of enrichment methods, comparing intrinsic and extrinsic partition of unity enrichment. Another review is provided in [1], which focuses on fracture simulations using partition of unity methods.
} 
the computational efficiency is improved by reducing the spatial dimension by one while the integration is efficient as well since no singular integration is introduced as in BEM. Natarajan et al. enhanced isogeometric analysis by the SBFEM, providing a method which inherits advantages of both FEM and IGABEM. Nevertheless, the approach does require a certain subdivision of the domain for complex geometries in order to obtain the scaling center [56].

\subsection{Dealing with component complexity}

Design cycle. Practical damage-tolerant engineering design requires typically a large number of iterations between designers on the one hand and stress and fatigue analysts on the other. The former group of engineers provide an initial design, which is refined iteratively through successive stress analyses and fracture propagation simulations under fatigue. The usual approach is to generate a mesh for stress analysis, compute the most highly stressed regions in the component and introduce a crack of dimensions defined by design codes at the location of maximum principle tensile stress in the component (this requires generating a new mesh, where the elements are typically several orders of magnitude smaller in size than the original stress analysis mesh). The crack is grown from this initial flaw and the number of cycles to fatigue is estimated. If the component's fatigue life happens to be unsatisfactory, the design is modified, and the whole process is repeated. It is clear that this approach is inefficient in terms of human intervention, slow, expensive and non-robust, since a typical design iteration can take as much as a few months for a relatively simple component.

Isogeometric analysis. Isogeometric analysis (IGA), first introduced by Hughes et al. [57] aims at enabling the engineer to use the same approximation functions to describe both the geometry of the component (provided by Computer Aided Design tools) and the solution of the problem (the displacement and stress fields in the present case). In its original, finite element based form, IGA does not completely suppress the need to create a mesh, as the CAD data provides only a 'shell' for the component, but gives no information about the parametrization of the interior of the volume which it encloses. Although the finite element version of IGA was used for fracture analysis [58-61]. This motivated the inception of a boundary element version of IGA for elasticity problems in [62-64].

Isogeometric boundary element methods: stress analysis directly from CAD. The conjoint use of IGA and BEM (IGABEM) permits the direct use of the CAD model, without any mesh generation and was developed for stress analysis [63,62,64], shape optimization [65-67]. This approach provides a tight and seamless transition between CAD and stress analysis. IGABEM has thus been subsequently extended to many fields [68-71]. PU enriched IGABEM was later proposed in [72,73] and trimmed NURBS approaches were presented in [74,75]. Accelerated solutions were proposed in [42] and Galerkin version of IGABEM in [76,77] etc. The benefit of smoothness to boundary integrals (BIEs) brought by IGA was recently investigated in [78]. Recently the isogeometric SGBEM has been applied for 2D fracture mechanics [79]. However, most work known to us primarily focuses on 2D problems, which are inconsequential for practical engineering applications. Indeed, the generation of analysis-suitable 3D volume parametrization for complex geometries is still an open question [80-82].

Based on the above discussion, the goal of our contribution is to investigate the advantages and drawbacks of isogeometric collocation boundary element methods for linear elastic fracture. In particular, we investigate:

1. How the higher-order continuity of the spline basis improves the accuracy of the stress field near the crack tip, crucial in fracture analysis and how this impacts the required number of degrees of freedom compared to a $C^{0}$ or discontinuous Lagrange basis;

2. The preservation and continuity of the curvature, tangential and normal vectors along the crack front during crack growth and evolved thanks to the exact representation of curved cracks;

3. The advantages of constructing a local crack front system directly based on the spline-based curve or surfacerepresented cracks and the impact of this direct construction on the accuracy of the fracture parameters;

4. The lack of volume parametrization/reparametrization for crack initiation and propagation. The cracks are modeled by spline surfaces directly as external boundaries of the geometry.

In short, we explore the qualities of IGABEM for 3D fracture analysis and fatigue crack growth. We use dual integral equations to model cracks, a local singularity removing technique proposed by Guiggiani [83] is applied on the various orders of singular integrals (up to hyper-singular $O\left(1 / r^{3}\right)$ ), and its improved version is tailored to handle distorted elements (or elements with high aspect ratio) which commonly arise in isogeometric based methods. The crack is 
a

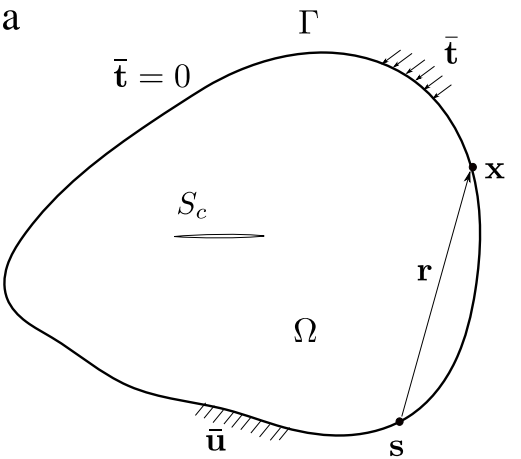

Fig. 1. Crack model. displacement BIE for $\mathbf{s}^{+}$

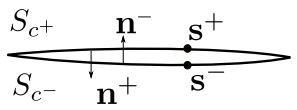

traction BIE for $\mathbf{s}^{-}$

explicitly represented by a NURBS surface as an internal boundary and an algorithm is outlined to describe the crack propagation such that the smoothness in geometry brought by IGA and in the solution brought by BEM is fully leveraged for extracting the stress intensity factors and simulating crack growth.

Outline of the paper. The rest of the paper is organized as follows. Section 2 briefs the boundary integral equations (BIEs) that applied in fracture modeling. Section 3 illustrates the NURBS basis functions on 2D surfaces and the collocation scheme. Section 4 outlines the improved singular integration based on the singularity subtraction technique [83]. The crack growth related work is detailed in Section 5, including updating the crack surface geometrically, computing the stress intensity factors and the fatigue fracture rule: the Paris law. Numerical examples for both static fracture analysis and crack growth are given in Section 6. We conclude our work and propose the future research of interest in the last section.

\section{Boundary integral equations for crack modeling}

Consider an arbitrary domain $\Omega$ which contains a crack as in Fig. 1. The boundary of the domain $\Gamma=S_{r} \cup S_{c^{+}} \cup S_{c^{-}}$, where the non-crack boundary $S_{r}$ is composed of $S_{u}$ where Dirichlet boundary conditions are prescribed (known displacements $\overline{\mathbf{u}}$ ), $S_{t}$ where Neumann boundary conditions are prescribed (known tractions $\overline{\mathbf{t}}$ ). The displacement BIE is given by finding $\mathbf{u}$ and $\mathbf{t}$ such that

$$
c_{i j}(\mathbf{s}) u_{j}(\mathbf{s})=\int_{\Gamma} U_{i j}(\mathbf{s}, \mathbf{x}) t_{j}(\mathbf{x}) \mathrm{d} S(\mathbf{x})-f_{\Gamma} T_{i j}(\mathbf{s}, \mathbf{x}) u_{j}(\mathbf{x}) \mathrm{d} S(\mathbf{x}),
$$

where the $U_{i j}, T_{i j}$ are called fundamental solutions and, for linear elasticity,

$$
\begin{aligned}
& U_{i j}(\mathbf{s}, \mathbf{x})=\frac{1}{16 \pi \mu(1-v) r}\left[(3-4 v) \delta_{i j}+r_{, i} r_{, j}\right], \\
& T_{i j}(\mathbf{s}, \mathbf{x})=-\frac{1}{8 \pi(1-v) r^{2}}\left\{\frac{\partial r}{\partial n}\left[(1-2 v) \delta_{i j}+3 r_{, i} r_{, j}\right]-(1-2 v)\left(r_{, i} n_{j}-r_{, j} n_{i}\right)\right\},
\end{aligned}
$$

where $\mu=E /[2(1+v)], E$ is Young's Modulus and $v$ Poisson's ratio. $\mathbf{s}$ is the source point (or collocation point. This two terms will be used interchangeably in the remainder part of this paper). $f$ denotes the integral is interpreted in the Cauchy Principal Value sense. The traction BIE is obtained by differentiation of the displacement BIE with respect to $\mathbf{s}$ and multiplication by the elastic tensor $E_{i j k l}$ :

$$
\begin{aligned}
& c_{i j}(\mathbf{s}) t_{j}(\mathbf{s})=f_{\Gamma} K_{i j}(\mathbf{s}, \mathbf{x}) t_{j}(\mathbf{x}) \mathrm{d} S(\mathbf{x})-f_{\Gamma} H_{i j}(\mathbf{s}, \mathbf{x}) u_{j}(\mathbf{x}) \mathrm{d} S(\mathbf{x}), \\
& H_{i j}(\mathbf{s}, \mathbf{x})=E_{i k p q} \frac{\partial T_{p j}(\mathbf{s}, \mathbf{x})}{\partial s_{q}} n_{k}(\mathbf{s}), \quad K_{i j}(\mathbf{s}, \mathbf{x})=E_{i k p q} \frac{\partial U_{p j}(\mathbf{s}, \mathbf{x})}{\partial s_{q}} n_{k}(\mathbf{s}),
\end{aligned}
$$

where $f$ denotes the Hadamard Finite Part integral. $c_{i j}$ is called a jump term and on a smooth boundary $c_{i j}=0.5 \delta_{i j}$. 
The idea of the boundary element method is to discretize the boundary geometry and the physical fields using sets of basis functions. Subsequently, the source point is placed at the collocation points and the displacement BIE in Eq. (1) or traction BIE (4) is transformed into a system of linear algebraic equations. However, when the domain contains a crack, the collocation points on the overlapping surfaces $S_{c^{+}}$and $S_{c^{-}}$could coincide (refer to Fig. 1(b)). The system matrix becomes then singular. Two ways to deal with this problem are given in the following sections.

\subsection{Dual equations}

The difficulty caused by the collapsed crack surfaces is circumvented through the use of dual equations, by prescribing different BIEs on either face of the crack. The displacement BIE (Eq. (1)) is used on one face $\left(S_{c^{+}}\right)$ and on the non-crack boundary $S_{r}$. The traction BIE (Eq. (4)) is used on the other crack face $\left(S_{c^{-}}\right)$. For the collocation point $\mathbf{s}^{+}$on the crack surface $S_{c^{+}}$, Eq. (1) can be rewritten as,

$$
\begin{aligned}
c_{i j}\left(\mathbf{s}^{+}\right) u_{j}\left(\mathbf{s}^{+}\right)+c_{i j}\left(\mathbf{s}_{m}^{-}\right) u_{j}\left(\mathbf{s}_{m}^{-}\right)= & \int_{S_{r}} U_{i j}\left(\mathbf{s}^{+}, \mathbf{x}\right) t_{j}(\mathbf{x}) \mathrm{d} S(\mathbf{x})-\int_{S_{r}} T_{i j}\left(\mathbf{s}^{+}, \mathbf{x}\right) u_{j}(\mathbf{x}) \mathrm{d} S(\mathbf{x}) \\
& -\int_{S_{c^{+}}} T_{i j}\left(\mathbf{s}^{+}, \mathbf{x}^{+}\right) u_{j}\left(\mathbf{x}^{+}\right) \mathrm{d} S(\mathbf{x})-\int_{S_{c^{-}}} T_{i j}\left(\mathbf{s}_{m}^{-}, \mathbf{x}^{-}\right) u_{j}\left(\mathbf{x}^{-}\right) \mathrm{d} S(\mathbf{x}) \\
& +\int_{S_{c^{+}}} U_{i j}\left(\mathbf{s}^{+}, \mathbf{x}^{+}\right) t_{j}\left(\mathbf{x}^{+}\right) \mathrm{d} S(\mathbf{x})+\int_{S_{c^{-}}} U_{i j}\left(\mathbf{s}_{m}^{-}, \mathbf{x}^{-}\right) t_{j}\left(\mathbf{x}^{-}\right) \mathrm{d} S(\mathbf{x}) .
\end{aligned}
$$

Analogously, the traction BIE (Eq. (4)) on the other crack surface $\left(S_{c^{-}}\right.$in Fig. 1(b)) becomes,

$$
\begin{aligned}
c_{i j}\left(\mathbf{s}^{-}\right) t_{j}\left(\mathbf{s}^{-}\right)+c_{i j}\left(\mathbf{s}_{m}^{+}\right) t_{j}\left(\mathbf{s}_{m}^{+}\right)= & \int_{S_{r}} K_{i j}\left(\mathbf{s}^{-}, \mathbf{x}\right) t_{j}(\mathbf{x}) \mathrm{d} S(\mathbf{x})-\int_{S_{r}} H_{i j}\left(\mathbf{s}^{-}, \mathbf{x}\right) u_{j}(\mathbf{x}) \mathrm{d} S(\mathbf{x}) \\
& -\oint_{S_{c^{-}}} H_{i j}\left(\mathbf{s}^{-}, \mathbf{x}^{-}\right) u_{j}\left(\mathbf{x}^{-}\right) \mathrm{d} S(\mathbf{x})-\oint_{S_{c^{+}}} H_{i j}\left(\mathbf{s}_{m}^{+}, \mathbf{x}^{+}\right) u_{j}\left(\mathbf{x}^{+}\right) \mathrm{d} S(\mathbf{x}) \\
& +\oint_{S_{c^{-}}} K_{i j}\left(\mathbf{s}^{-}, \mathbf{x}^{-}\right) t_{j}\left(\mathbf{x}^{-}\right) \mathrm{d} S(\mathbf{x})+f_{S_{c^{+}}} K_{i j}\left(\mathbf{s}_{m}^{+}, \mathbf{x}^{+}\right) t_{j}\left(\mathbf{x}^{+}\right) \mathrm{d} S(\mathbf{x}) .
\end{aligned}
$$

$\mathbf{s}_{m}^{-}$denotes the mirror point of $\mathbf{s}^{+}$on $S_{c^{-}}$, which means $\mathbf{s}_{m}^{-}$and $\mathbf{s}^{-}$share the same physical and parametric coordinates but their normal vectors are opposite. The last two terms of both equations and left hand side of Eq. (7) are omitted due to the traction-free crack.

Remark. Due to the collapsed boundary, two jump terms arise in each BIE and each operator not only exhibits singularity on the crack surface where the collocation points are located, but also on the one where the mirror points of the collocation points are located.

\subsection{Crack opening displacement (COD) equation}

The boundary integral equation for crack problem can also be reformulated by setting the boundary quantity as crack opening displacement over a couple of crack surfaces. Let the source point approach one crack surface, for example $S_{c}=S_{c^{+}}$, and note that $\mathbf{n}=\mathbf{n}^{+}=-\mathbf{n}^{-}$, we have:

$$
\begin{aligned}
c_{i j}\left(\mathbf{s}^{+}\right) u_{j}\left(\mathbf{s}^{+}\right)+c_{i j}\left(\mathbf{s}_{m}^{-}\right) u_{j}\left(\mathbf{s}_{m}^{-}\right)= & \int_{S_{r}} U_{i j}\left(\mathbf{s}^{+}, \mathbf{x}\right) t_{j}(\mathbf{x}) \mathrm{d} S(\mathbf{x})-f_{S_{r}} T_{i j}\left(\mathbf{s}^{+}, \mathbf{x}\right) u_{j}(\mathbf{x}) \mathrm{d} S(\mathbf{x}) \\
& +\int_{S_{c}} U_{i j}\left(\mathbf{s}^{+}, \mathbf{x}^{+}\right)\left(t_{j}\left(\mathbf{x}^{+}\right)+t_{j}\left(\mathbf{x}^{-}\right)\right) \mathrm{d} S(\mathbf{x}) \\
& -\int_{S_{c}} T_{i j}\left(\mathbf{s}^{+}, \mathbf{x}^{+}\right)\left(u_{j}\left(\mathbf{x}^{+}\right)-u_{j}\left(\mathbf{x}^{-}\right)\right) \mathrm{d} S(\mathbf{x}) .
\end{aligned}
$$


The corresponding traction BIE is:

$$
\begin{aligned}
c_{i j}\left(\mathbf{s}^{+}\right) t_{j}\left(\mathbf{s}^{+}\right)+c_{i j}\left(\mathbf{s}_{m}^{-}\right) t_{j}\left(\mathbf{s}_{m}^{-}\right)= & f_{S_{r}} K_{i j}\left(\mathbf{s}^{+}, \mathbf{x}\right) t_{j}(\mathbf{x}) \mathrm{d} S(\mathbf{x})-f_{S_{r}} H_{i j}\left(\mathbf{s}^{+}, \mathbf{x}\right) u_{j}(\mathbf{x}) \mathrm{d} S(\mathbf{x}) \\
& +f_{S_{c}} K_{i j}\left(\mathbf{s}^{+}, \mathbf{x}^{+}\right)\left(t_{j}\left(\mathbf{x}^{+}\right)+t_{j}\left(\mathbf{x}^{-}\right)\right) \mathrm{d} S(\mathbf{x}) \\
& -f_{S_{c}} H_{i j}\left(\mathbf{s}^{+}, \mathbf{x}^{+}\right)\left(u_{j}\left(\mathbf{x}^{+}\right)-u_{j}\left(\mathbf{x}^{-}\right)\right) \mathrm{d} S(\mathbf{x}) .
\end{aligned}
$$

Eq. (9) can be used alone if the COD alone will be used as the unknown for the fatigue crack growth problem. However if the displacement field needs to be known on the crack surfaces, Eq. (8) should also be solved. For a infinite domain $\left(S_{r} \rightarrow \infty\right)$, assuming that traction-free crack faces are assumed, we arrive at:

$$
0=t_{j}^{\infty}(\mathbf{s})-f_{S_{c}} H_{i j}(\mathbf{s}, \mathbf{x}) \llbracket u_{j}(\mathbf{x}) \rrbracket \mathrm{d} S(\mathbf{x}) .
$$

$\llbracket u_{j}(\mathbf{x}) \rrbracket=u_{j}\left(\mathbf{x}^{+}\right)-u_{j}\left(\mathbf{x}^{-}\right)$is the crack opening displacement. All the subscripts ' + ' are omitted since the integral is only over a single crack surface. $\mathbf{t}^{\infty}$ is interpreted as the solution in the 'no crack' space.

\section{NURBS discretization and collocation}

NURBS basis functions are a generalization of B-spline functions that allows a 'projection' from square and cubic domains to form general geometries. B-spline basis functions are defined over a knot vector, which is a non-decreasing sequence of real numbers given in the parameter space. A knot vector is denoted as $\Xi=\left\{\xi_{1}, \xi_{2}, \ldots, \xi_{n+p+1}\right\}$, where $\xi_{i} \in \mathbb{R}$ is the $i$ th parameter coordinate (knot), $p$ is the order of the polynomial, $n$ is the number of the basis functions. For a given order $p$, the B-spline basis functions $N_{i, p}$ with $1 \leqslant a \leqslant n$ are defined by the Cox-de Boor recursion:

$$
N_{i, 0}(\xi)= \begin{cases}1 & \xi_{i} \leqslant \xi<\xi_{i+1} \\ 0 & \text { otherwise }\end{cases}
$$

then, for $p>0$,

$$
N_{i, p}(\xi)=\frac{\xi-\xi_{i}}{\xi_{i+p}-\xi_{i}} N_{i, p-1}(\xi)+\frac{\xi_{i+p+1}-\xi}{\xi_{i+p+1}-\xi_{i+1}} N_{i+1, p-1}(\xi)
$$

The continuity of B-spline basis functions at $\xi_{i}$ can be decreased by repeating the knot several times. If $\xi_{i}$ has multiplicity $k\left(\xi_{i}=\xi_{i+1}=\cdots=\xi_{i+k-1}\right)$, then the basis functions are $C^{p-k}$ continuous at $\xi_{i}$. Particularly, when $k=p$, the basis is $C^{0}$ and $k=p+1$ leads to a discontinuity at $\xi_{i}$. If the first and last knot have $k=p+1$, the knot vector is called an open knot vector. More details can be found in $[84,60]$.

Given the knot vectors $\Xi=\left\{\xi_{1}, \xi_{2}, \ldots, \xi_{n+p+1}\right\}$ and $\mathscr{H}=\left\{\eta_{1}, \eta_{2}, \ldots, \eta_{m+q+1}\right\}$, and the control points net $P_{i, j}$. The B-spline surface $S(\xi, \eta)$ is given by the tensor-product of B-spline basis functions defined in the 2D parametric domain $\left[\xi_{1}, \xi_{n+p+1}\right] \times\left[\eta_{1}, \eta_{m+q+1}\right]$,

$$
S(\xi, \eta)=\sum_{i=1}^{n} \sum_{j=1}^{m} N_{i, p}(\xi) M_{j, q}(\eta) P_{i, j},
$$

where $N_{i, p}(\xi) M_{j, q}(\eta)$ are the $2 \mathrm{D}$ B-spline basis functions. The NURBS basis functions can be constructed by rationalizing the tensor-product B-spline basis functions as

$$
R_{i, j}(\xi, \eta)=\frac{N_{i, p}(\xi) M_{j, q}(\eta) w_{i, j}}{\sum_{k=1}^{n} \sum_{l=1}^{m} N_{k, p}(\xi) M_{l, q}(\eta) w_{k, l}},
$$

where the scalar variable $w_{i, j}$ is the weight associated with the control point $P_{i, j}$. For the sake of integration, the 2D NURBS basis functions are usually calculated in the element defined by the non-zero knot intervals $\left[\xi_{i}, \xi_{i+1}\right] \times\left[\eta_{j}, \eta_{j+1}\right]$ where the Gaussian rule can be applied [57]. 
In the NURBS based isogeometric concept, the physical field is approximated by the same NURBS basis functions as those used to describe the geometry $\Gamma=S_{r}(\xi, \eta) \cup S_{c^{+}}(\xi, \eta) \cup S_{c^{-}}(\xi, \eta)=\cup_{e=1}^{N_{e}} S_{e}(\xi, \eta), N_{e}$ is the number of NURBS elements. The displacement and traction fields can be approximated as follows:

$$
\begin{aligned}
& u_{i}(\xi, \eta)=\sum_{A=1}^{n} R_{A}(\xi, \eta) d_{i}^{A}, \\
& t_{i}(\xi, \eta)=\sum_{A=1}^{n} R_{A}(\xi, \eta) q_{i}^{A},
\end{aligned}
$$

where $n=(p+1)(q+1)$ is the number of NURBS basis functions for an element and the construction of the map between the global index $A$ and the local index $(i, j)$ can be found in [57]. And $d_{i}, q_{i}$ are displacement and traction control variables respectively. The parameter domain of an element $\left[\xi_{i}, \xi_{i+1}\right] \times\left[\eta_{j}, \eta_{j+1}\right]$ is converted into $[-1,1] \times[-1,1]$, which is called the parent space. We define $\xi\left(\xi_{i}=\xi, \eta\right)$ as the parameter coordinate and $\bar{\xi}\left(\bar{\xi}_{i}=\bar{\xi}, \bar{\eta}\right)$ as the parent coordinate of the field point $\mathbf{x}$, and $\bar{J}=\bar{J}(\bar{\xi}, \bar{\eta})$ is the Jacobian transformation from physical to parent space. The transformation for a NURBS element from parameter to parent space is

$$
\begin{aligned}
\xi & =\frac{\left(\xi_{i+1}-\xi_{i}\right) \bar{\xi}+\left(\xi_{i+1}+\xi_{i}\right)}{2}, \\
\eta & =\frac{\left(\eta_{i+1}-\eta_{i}\right) \bar{\eta}+\left(\eta_{i+1}+\eta_{i}\right)}{2}, \\
\bar{J} & =\frac{\mathrm{d} S}{\mathrm{~d} \xi} \frac{\left(\xi_{i+1}-\xi_{i}\right)}{2}+\frac{\mathrm{d} S}{\mathrm{~d} \eta} \frac{\left(\eta_{i+1}-\eta_{i}\right)}{2},
\end{aligned}
$$

where $\frac{\mathrm{d} S}{\mathrm{~d} \xi}$ and $\frac{\mathrm{d} S}{\mathrm{~d} \eta}$ are calculated from Eq. (13). Substituting the discretized displacements and tractions into the BIEs (6) and (7) will give,

$$
\begin{aligned}
\sum_{I=1}^{n} C_{i j}^{I}\left(\mathbf{s}^{+}\right) d_{j}^{+, I}+\sum_{I=1}^{n} C_{i j}^{I}\left(\mathbf{s}_{m}^{-}\right) d_{j}^{-, I}= & \sum_{S_{e} \subset S_{r}} \sum_{I=1}^{n} U_{i j}^{I} q_{j}^{I}-\sum_{S_{e} \subset S_{r}} \sum_{I=1}^{n} T_{i j}^{I} d_{j}^{I} \\
& -\sum_{S_{e} \subset S_{c^{+}}} \sum_{I=1}^{n} T_{i j}^{I} d_{j}^{+, I}-\sum_{S_{e} \subset S_{c^{-}}} \sum_{I=1}^{n} T_{i j}^{I} d_{j}^{-, I} \\
& +\sum_{S_{e} \subset S_{c^{+}}} \sum_{I=1}^{n} U_{i j}^{I} q_{j}^{+, I}+\sum_{S_{e} \subset S_{c^{-}}} \sum_{I=1}^{n} U_{i j}^{I} q_{j}^{-, I} \\
\sum_{I=1}^{n} C_{i j}^{I}\left(\mathbf{s}^{-}\right) q_{j}^{-, I}+\sum_{I=1}^{n} C_{i j}^{I}\left(\mathbf{s}_{m}^{+}\right) q_{j}^{+, I}= & \sum_{S_{e} \subset S_{r}} \sum_{I=1}^{n} K_{i j}^{I} q_{j}^{I}-\sum_{S_{e} \subset S_{r}} \sum_{I=1}^{n} H_{i j}^{I} d_{j}^{I} \\
& -\sum_{S_{e} \subset S_{c^{-}}} \sum_{I=1}^{n} H_{i j}^{I} d_{j}^{-, I}-\sum_{S_{e} \subset S_{c^{+}}} \sum_{I=1}^{n} H_{i j}^{I} d_{j}^{+, I} \\
& +\sum_{S_{e} \subset S_{c^{-}}} \sum_{I=1}^{n} K_{i j}^{I} q_{j}^{-, I}+\sum_{S_{e} \subset S_{c^{+}}} \sum_{I=1}^{n} U_{i j}^{I} q_{j}^{+, I}
\end{aligned}
$$

where the jump term and integrals of the fundamental solutions are respectively written as:

$$
\begin{aligned}
& C_{i j}^{I}(\mathbf{s})=c_{i j} R_{I}\left(\boldsymbol{\xi}_{s}\right), \\
& F_{i j}^{I}=\int_{S_{e}} F_{i j}(\mathbf{s}, \mathbf{x}(\boldsymbol{\xi})) R_{I}(\boldsymbol{\xi}) \bar{J}(\bar{\xi}) \mathrm{d} S, \quad F \text { can be } T, U, H, K .
\end{aligned}
$$

Note that the discretization process of the singular integrals will lead to singular elements $\left(s \in S_{e}\right)$ and non-singular elements $\left(s \notin S_{e}\right.$ ). The numerical quadrature for them needs to be treated separately. Let us take the kernel $H$ with 
hyper-singular term for example, if $\mathbf{s}=\mathbf{s}^{+} \in S_{e 0}^{+}$:

$$
\begin{aligned}
& H_{i j}^{I}=f_{S_{e}} H_{i j}\left(\mathbf{s}^{+}, \mathbf{x}^{+}(\boldsymbol{\xi})\right) R_{I}(\xi) J(\bar{\xi}) \mathrm{d} S, \quad \text { if } S_{e}=S_{e 0}^{+} ; \\
& H_{i j}^{I}=f_{S_{e}} H_{i j}\left(\mathbf{s}_{m}^{-}, \mathbf{x}^{-}(\boldsymbol{\xi})\right) R_{I}(\xi) J(\bar{\xi}) \mathrm{d} S, \quad \text { if } S_{e}=S_{m, e 0}^{-}, \text {the mirror element of } S_{e 0}^{+} ; \\
& H_{i j}^{I}=\int_{S_{e}} H_{i j}\left(\mathbf{s}^{+}, \mathbf{x}(\boldsymbol{\xi})\right) R_{I}(\boldsymbol{\xi}) J(\bar{\xi}) \mathrm{d} S, \quad \text { if } S_{e} \subset \Gamma-S_{e 0}^{+}-S_{m, e 0}^{-} .
\end{aligned}
$$

The discretized COD equation (10) can be obtained in a similar way and its implementation is simpler as only one crack face is used and the singularity in the 'mirror' element does not appear.

The Greville abscissae (GA) has been used to generate the collocation points. In IGA, the number of control points in the geometry representation has to be equal to the number of control variables in the field approximation, which requires the same number of collocation points. For domains with continuous parametrization, composed by trimless and compatible NURBS patches, the number of collocation points obtained by the Greville abscissae is equal to the number of control points in the geometry representation, which is suitable for approximation of continuous solutions. However, in some cases the collocation points, generated by the GA may not be suitable for the analysis. For example, for the geometries with sharp edges or corners, the discontinuous tractions can be better approximated by the discontinuous basis functions, which can be obtained from the original geometry parametrization by doubling the knot values and the corresponding control points. Or for the geometries parametrized by degenerated elements, when the number of the collocation points generated by the GA does not match the number of control variables in the solution approximation. In order to match the number of discontinuous basis functions, the collocation points are shifted inside an element by an offset $\alpha$ by:

$$
\begin{array}{ll}
\xi_{s, i}=\xi_{s, i}+\alpha\left(\xi_{s, i+1}-\xi_{s, i}\right), & \text { or } \\
\xi_{s, i}=\xi_{s, i}-\alpha\left(\xi_{s, i}-\xi_{s, i-1}\right), & 0<\alpha<1 .
\end{array}
$$

In this work, the knot values and control variables are doubled in order to approximate the traction fields. While flexible choices can be made to perform more efficient mesh refinement related to the geometry independent field approximation, see [42]. We take $\alpha=0.5$ without particularly specified. We will show in the numerical example that the closer to 0 the value of $\alpha$ is, the more accurate the approximation will be. However, a smaller $\alpha$ will evoke stronger 'near-singularity' in the neighborhood elements. Further improvements to overcome this difficulty will be pursued in the future work.

\section{Numerical integration}

Due to the singularities in BIEs, there will be singular integration and non-singular integration after discretization. For the element containing the collocation point, singular integration is performed and in view of this, the element is called 'singular'. Elements which exclude the collocation point are called non-singular elements. Singular integration needs to be carefully treated in BEM. Various numerical methods have been proposed, and one can refer to a review work in [39]. A robust technique developed in [41] can be applied to regularize all the singular terms, i.e. transform them into weakly singular terms via the use of simple solution of BIE. The regularization technique based on simple solutions has been further developed in the framework of IGABEM [68,85,78]. However, this method fails when dealing with open surfaces such as cracks because of the existence of two jump terms in collapsed boundary [86]. In the present work, we use the singularity subtraction technique (SST) proposed by Guiggiani [86,83] to remove the singularities arising in both BIEs. The SST is a method for the treatment of singular integrals regardless of mesh discretization and proved to be efficient for fracture via dual BEM [44].

\subsection{Singularity subtraction technique (SST) for singular integrals}

The SST transforms various orders of singular integration into a weakly singular one based on the intrinsic coordinate system of the singular element after discretization. Then the weakly singular integration becomes regular if the quadrature is performed in polar coordinates. By expanding the integrand into a series with respect to the intrinsic 


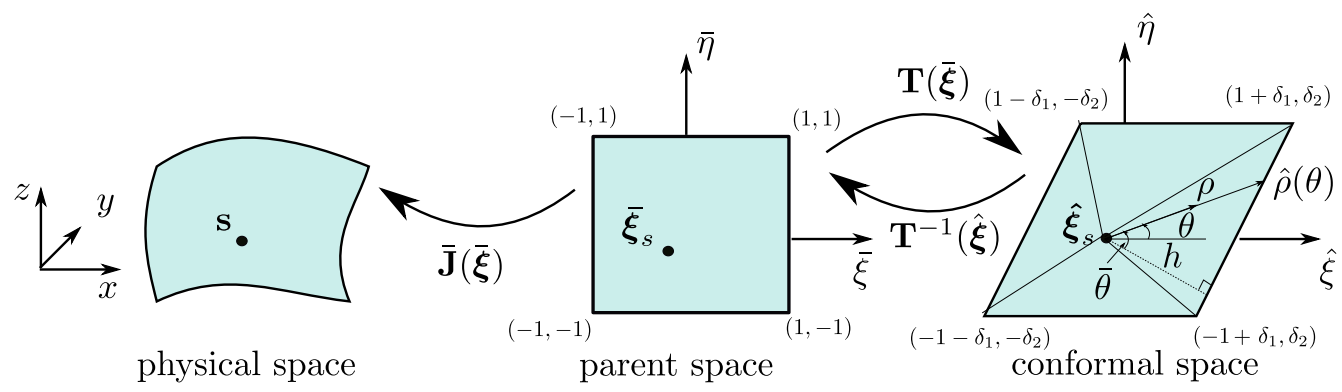

Fig. 2. Transformation between coordinate system for SST.

coordinates, the singularity can be represented explicitly with respected to the parametric distance between the source point and field point $\rho$. Then the singular terms are subtracted from the integrand, leaving the remaining to be regular for which regular Gaussian rule is applied. The subtracted terms are added back semi-analytically. For example, for the hyper-singular integral of the form

$$
I=\oint_{S_{e}} H(\mathbf{s}, \mathbf{x}(\boldsymbol{\xi})) R(\boldsymbol{\xi}) \bar{J}(\bar{\xi}) \mathrm{d} S,
$$

the polar coordinates $\rho(\rho, \theta)$ centered at the source point are introduced in the parent space (Fig. 2). The parent domain is subdivided into triangles for quadrature. For each field point $\bar{\xi}$ in the sub-triangles, we have

$$
\begin{aligned}
& \bar{\xi}=\bar{\xi}_{s}+\rho \cos \theta, \\
& \bar{\eta}=\bar{\eta}_{s}+\rho \sin \theta .
\end{aligned}
$$

After the polar coordinate transformation, Eq. (24) becomes

$$
I=\lim _{\varepsilon \rightarrow 0} \int_{0}^{2 \pi} \int_{\alpha(\varepsilon, \theta)}^{\hat{\rho}(\theta)} H(\rho, \theta) R(\rho, \theta) \bar{J}(\rho, \theta) \rho \mathrm{d} \rho \mathrm{d} \theta,
$$

where $\hat{\rho}(\theta)=h / \cos \bar{\theta} . h$ is the shortest distance from the source point to the element edge and $\bar{\theta}$ is the angle from perpendicular direction to the field point as in Fig. 2. If we define $\theta_{0}$ as the angle of the perpendicular line, then the angle to the field point can be computed as

$$
\theta=\bar{\theta}+\theta_{0} .
$$

The integrand $F(\rho, \theta)=H(\rho, \theta) R(\rho, \theta) \bar{J}(\rho, \theta) \rho$ is expanded as:

$$
F(\rho, \theta)=\frac{F_{-2}(\theta)}{\rho^{2}}+\frac{F_{-1}(\theta)}{\rho}+F_{0}(\theta)+F_{1}(\theta) \rho+F_{2}(\theta) \rho^{2}+\cdots=\sum_{i=-1}^{\infty} F_{i}(\theta) \rho^{i} .
$$

The first two singular terms on the right hand side are subtracted and added back semi-analytically, resulting in:

$$
\begin{aligned}
I & =I_{1}+I_{2}, \\
I_{1} & =\int_{0}^{2 \pi} \int_{0}^{\hat{\rho}(\theta)}\left[F(\rho, \theta)-\frac{F_{-2}(\theta)}{\rho^{2}}-\frac{F_{-1}(\theta)}{\rho}\right] \mathrm{d} \rho \mathrm{d} \theta, \\
I_{2} & =\int_{0}^{2 \pi} I_{-1}(\theta) \ln \frac{\hat{\rho}(\theta)}{\beta(\theta)} \mathrm{d} \theta-\int_{0}^{2 \pi} I_{-2}(\theta)\left[\frac{\gamma(\theta)}{\beta^{2}(\theta)}+\frac{1}{\hat{\rho}(\theta)}\right] \mathrm{d} \theta,
\end{aligned}
$$

where $I_{1}$ is regular and $I_{2}$ are regular line integrals, Both can be applied with Gaussian quadrature rule. The evaluation of $\alpha(\varepsilon, \theta), \beta(\theta)$ and $\gamma(\theta)$ as well as the limiting process can be found in [83]. 


\subsection{Conformal mapping for SST}

It has been revealed by Rong et al. [87] that the expansion coefficients $F_{i}(\theta)$ in Eq. (28) exhibits various orders of near-singularity in the angular $\theta$ direction, although the singularity in the radial $\rho$ direction disappears. This nearsingularity is sensitive to the shape of the element and becomes severe when the element is highly distorted. The $F_{i}(\theta)$ can be represented as [87]:

$$
F_{i}(\theta)=\frac{\tilde{F}_{i}(\theta)}{A^{p}(\theta)}=\frac{\tilde{F}_{i}(\theta)}{\left[0.5\left(\left|\mathbf{m}_{1}^{s}\right|^{2}+\left|\mathbf{m}_{2}^{s}\right|^{2}\right)(\omega \sin (2 \theta+\varphi)+1)\right]^{p / 2}},
$$

where $\tilde{F}_{i}(\theta)$ are the regular trigonometric functions and integer ' $p$ ' is the order associated with ' $i$ '. The curvilinear basis vectors $\mathbf{m}_{i}^{s}=\left.\mathbf{m}_{i}\right|_{\bar{\xi}=\bar{\xi}},(i=1,2)$ are calculated as:

$$
\begin{aligned}
& \mathbf{m}_{1}=\left[\frac{\partial x}{\partial \bar{\xi}}, \frac{\partial y}{\partial \bar{\xi}}, \frac{\partial z}{\partial \bar{\xi}}\right], \\
& \mathbf{m}_{2}=\left[\frac{\partial x}{\partial \bar{\eta}}, \frac{\partial y}{\partial \bar{\eta}}, \frac{\partial z}{\partial \bar{\eta}}\right] .
\end{aligned}
$$

We introduces two parameters

$$
\begin{aligned}
\lambda & =\left|\mathbf{m}_{1}^{s}\right| /\left|\mathbf{m}_{2}^{s}\right|, \\
\cos \psi & =\mathbf{m}_{1}^{s} \cdot \mathbf{m}_{2}^{s} /\left|\mathbf{m}_{1}^{s}\right|\left|\mathbf{m}_{2}^{s}\right|,
\end{aligned}
$$

such that

$$
\begin{aligned}
\varphi & =\arctan \frac{\lambda^{2}-1}{2 \lambda \cos \psi}, \\
\omega & =\sqrt{1-\frac{4 \sin ^{2} \psi}{\left(\lambda+\lambda^{-1}\right)^{2}}}<1 .
\end{aligned}
$$

It can be concluded that when the element aspect ratio is large or the angle between two basis vectors tends to 0 or $\pi$ ( $\sin \psi \rightarrow 0$ ), $A(\theta)$ will tend to 0 , resulting in the near-singularity of $F_{i}(\theta)$. Both scenarios indicate a distorted shape of the singular element, which are common in isogeometric analysis.

Rong et al. [87] constructed the conformal mapping from the parent space $(\bar{\xi}, \bar{\eta})$ to a new parametric space $(\hat{\xi}, \hat{\eta})$ where the two curvi-linear basis vectors in the new parametric space are orthogonal and have identical length, i.e.

$$
\begin{gathered}
\hat{\mathbf{m}}_{1}^{s} \cdot \hat{\mathbf{m}}_{2}^{s}=0, \\
\left|\hat{\mathbf{m}}_{1}^{s}\right|=\left|\hat{\mathbf{m}}_{2}^{s}\right| .
\end{gathered}
$$

Then $A(\theta)$ becomes a constant, which makes the integration nonsensitive to the element shape, if the series is expanded in the new space. The quadrature for the singular integral turns to be stable regardless of mesh distortion.

The mapping proposed by Rong et al. is tailored for triangular element, in this work we extend it to the quadrilateral elements (Fig. 2). In [87], the Jacobian transformation matrix $\mathbf{T}$ from $\bar{\xi}=(\bar{\xi}, \bar{\eta})$ to a new parametric space $\hat{\xi}=(\hat{\xi}, \hat{\eta})$ is

$$
\mathbf{T}=\left[\begin{array}{ll}
1 & \delta_{1} \\
0 & \delta_{2}
\end{array}\right], \quad \text { so that } \hat{\boldsymbol{\xi}}=\mathbf{T} \overline{\boldsymbol{\xi}}
$$

where $\delta_{1}=\cos \psi / \lambda, \delta_{2}=\sin \psi / \lambda$. Then the new basis vectors

$$
\left[\begin{array}{ll}
\hat{\mathbf{m}}_{1}^{s} & \hat{\mathbf{m}}_{2}^{s}
\end{array}\right]=\left[\begin{array}{ll}
\mathbf{m}_{1}^{s} & \mathbf{m}_{2}^{s}
\end{array}\right] \mathbf{T}^{-1}=\left[\begin{array}{ll}
\mathbf{m}_{1}^{s} & -\left(\delta_{1} / \delta_{2}\right) \mathbf{m}_{1}^{s}+\left(1 / \delta_{2}\right) \mathbf{m}_{2}^{s}
\end{array}\right]
$$


will satisfy the relation in Eqs. (34). The bilinear interpolation is used from $(\bar{\xi}, \bar{\eta})$ to the new parametric space $(\hat{\xi}, \hat{\eta})$ for a quadrilateral element:

$$
\begin{aligned}
\hat{\xi} & =\sum_{I=1}^{4} N_{I}(\bar{\xi}) \hat{\xi}^{I}, \\
N_{1}(\bar{\xi}, \bar{\eta}) & =0.25(1+\bar{\xi})(1+\bar{\eta}), \\
N_{2}(\bar{\xi}, \bar{\eta}) & =0.25(1-\bar{\xi})(1+\bar{\eta}), \\
N_{3}(\bar{\xi}, \bar{\eta}) & =0.25(1-\bar{\xi})(1-\bar{\eta}), \\
N_{4}(\bar{\xi}, \bar{\eta}) & =0.25(1+\bar{\xi})(1-\bar{\eta}) .
\end{aligned}
$$

Combining Eqs. (35) and (37), the nodal coordinates $\hat{\xi}^{I}$ can be obtained as $\hat{\xi}^{1}\left(1+\delta_{1}, \delta_{2}\right), \hat{\xi}^{2}\left(-1+\delta_{1}, \delta_{2}\right)$, $\hat{\xi}^{3}\left(-1-\delta_{1},-\delta_{2}\right)$ and $\hat{\xi}^{4}\left(1-\delta_{1},-\delta_{2}\right)$. It should be noted that since $0<\psi<\pi, \delta_{2}>0$, the quadrilateral element is guaranteed to have positive area (one possible plot is shown in Fig. 2). This requires the source point to be located at the degenerated point in the geometry where $\left|\mathbf{m}_{i}^{s}\right| \neq 0$.

It can be seen from Fig. 2 that the shape of the element in conformal space is controlled by the coefficients $\delta_{1}$ and $\delta_{2}$. This means that if $\lambda$ (reflecting the element aspect ratio) and $\cos \psi$ (reflecting the element distortion) deviate from 1 , the conformal element will be skewed. This will result in sub-triangles with $\bar{\theta}$ approaching $\pm \pi / 2$ if the field point is close to the edges adjacent to the source point of the sub-triangles (Fig. 2). Thus $\hat{\rho}(\theta)=h / \cos \bar{\theta}$ is not calculated accurately. To alleviate this near singularity in $\hat{\rho}(\theta)$, the following Sigmoidal transformation is applied in the angular direction such that the integration points will be adaptively clustered close to the edges where the near-singularity is severe, according to the value of $\bar{\theta}$ [87],

$$
\begin{aligned}
w(\bar{\theta}) & =\frac{1}{\pi}\left(\bar{\theta}+\frac{\pi}{2}\right), \quad-\frac{\pi}{2}<\bar{\theta}<\frac{\pi}{2}, 0<z<1, \\
z & =z(s)=w\left(\bar{\theta}_{1}\right)+\frac{1}{2}(s+1)\left(w\left(\bar{\theta}_{2}\right)-w\left(\bar{\theta}_{1}\right)\right), \quad-1<s<1,0 \leq z\left(\bar{\theta}_{1}\right)<z<z\left(\bar{\theta}_{2}\right) \leq 1 \\
f(z) & =\frac{z^{m}}{z^{m}+(1-z)^{m}}, \\
\bar{\theta} & =\pi f(z)-\frac{\pi}{2} \\
J^{-1}(\theta) & =\frac{\partial \theta}{\partial s}=\frac{\pi\left[w\left(\bar{\theta}_{2}\right)-w\left(\bar{\theta}_{1}\right)\right] m f(z)^{m-1}}{2\left(f(z)^{m}+(1-f(z))^{m}\right)^{2}},
\end{aligned}
$$

where $s$ is the Gauß point from interval $(-1,1)$, the relation of $\theta$ and $\bar{\theta}$ can be found in Eq. (27).

\subsection{Numerical quadrature}

In our numerical implementation, a Gaussian rule is applied in both radial and angular direction. Combining with the study in [87] and our numerical tests of singular integration for NURBS elements (which are not presented in the paper for brevity), we use $6 \mathrm{Gau} ß$ points are used in the radial direction, $18 \mathrm{Gau} ß$ points in the angular direction of each sub-triangle for conformal SST unless otherwise specified. For each non-singular element, an adaptive subdivision scheme is used according to the relative distance between the element and collocation point. All the rules are used empirically without any error control algorithm.

\section{Crack growth}

The approaches used to represent and track the crack propagation can be classified into two manifolds, implicit and explicit methods. A typical application of the former method would be the level set method [88] which was coupled in the XFEM/GFEM to represent and grow the discontinuity [30,31]. The level set function is a signed distance function to the crack surface defined on the underlying mesh, which could be either consistent with the mesh discretization 
of the problem or an independent structured mesh. Since the cracks are open surfaces, one more level set function, perpendicular to the crack surface is required in order to describe the crack front. The quality to represent the crack surface depends on the resolution of the underlying mesh. Accurately describing the crack surface usually introduces additional computational expense [89]. Advection-type equations should be solved so as to update the crack front when the crack evolves [90] which increases the computational effort. Chopp and Sukumar [91] proposed the fast marching method to update the crack front, thus facilitating the process of updating the crack surface [29]. Fries and Baydoun [92] proposed an implicit-explicit method, in which the level set represented crack is explicitly discretized by triangular facets. Analogous idea is the vector level set method [35]. These methods take advantage of the level set representation for the PU enrichment while avoiding to update the crack surface by solving the equations. Additionally, sharp turns and kinks can be retained by use of explicit crack surfaces rather than pure level sets.

The explicit method uses sets of triangular or quadrilateral facets to discretize the crack surface directly. For finite element based methods, the crack evolution process is usually accompanied with an automatic re-meshing operation. For XFEM/GFEM applications, the subdivision of the 3D solid elements needs to be performed for the integration purpose. Both relies on well-developed meshing/re-meshing packages [93,94]. The explicit representation of the crack surfaces by triangulation has been used in meshfree methods as well [34,1,36]. It should be noted that this representation method usually results in a $C^{0}$ crack surface and that the crack fronts are composed of line segments. This leads to at least two shortcomings: (1) The crack front is not smooth which leads to inaccuracies in the extraction of the fracture parameters (for example the SIFs). Geometry approximation error accumulates as the crack grows; (2) The local crack front coordinate system is not well defined and the deflection angle is discontinuous, resulting in non-unique branch enrichment values for some points on the crack front. One approach to alleviate this is to abandon the branch enrichment $[1,36]$. As a remedy, the crack fronts need to be smoothed through some numerical techniques [93,94]. Similar scenarios occur in Lagrange based BEM for fracture modeling. Besides, Paluszny and Zimmerman [95] point out that a large number of facets are needed in order to more accurately represent the crack surface so that the storage increases rapidly with respect to the area of the crack surface when the cracks propagate. Hence they propose the use of a parametric surface, i.e. the NURBS patch, to describe the crack propagation. In their approach, crack growth is realized geometrically by deforming the NURBS surface through the mid-range LaGreca algorithm [96] to move the control points. Due to the parametrization of the NURBS patch, the crack tip can be sampled anywhere along the crack front, thus the storage for crack discretization increases mildly. Meanwhile the local crack front coordinate system is established on the smooth geometry. However, this method is based on re-meshing the finite elements. Recently Tambat et al. proposed an explicitly represented lower-dimension geometry features by NURBS $[97,98]$ through the partition of unity approximation. Instead of using level sets, the lower-dimension features such as cracks are accurately described through the calculation of the distance field in an efficient noniterative way, providing a promising alternative to evolve discontinuities in the IGAFEM framework. However, more suitable numerical quadrature scheme are still desired in order to fully exploit the exact representation in geometry.

In the present work, we use NURBS patches to discretize the crack surfaces. The crack front is exactly described and the local crack tip system is defined naturally and uniquely based on the NURBS patch. Meanwhile, the physical quantities are also approximated by the NURBS basis in the spirit of isogeometric analysis. With BEM, the smoothness in geometry and stress solution is fully exploited to calculate the fracture parameters and evolve the cracks. In the following section, we first introduce the ways to extract the stress intensity factors, based on the NURBS crack surfaces and recall the Paris law to calculate the crack advance. With these governing fracture parameters, a crack surface updating algorithm is outlined to perform the crack growth.

\subsection{Computation of stress intensity factors}

The driving force for the evolution of fatigue fracture is characterized by some fracture parameters such as the stress intensity factors (SIFs), which can be extracted from the numerical solution. If the fracture parameters are computed based on the point-wise tips on the crack front independently, the approach can be considered as 'local'. The key factor to compute accurately the SIFs in a local approach is to avoid discretization and path dependence.

Various methods have been developed to extract the SIFs in the framework of FEM and BEM. The displacement correlation method [99], with or without crack-tip singular elements, is simple and fast. Nevertheless, this method is extraction path dependent. An extrapolation technique is typically performed upon a group of calculations to avoid such dependence. The virtual crack extension method (VCE), was applied to compute SIFs in [100,101]. The original 


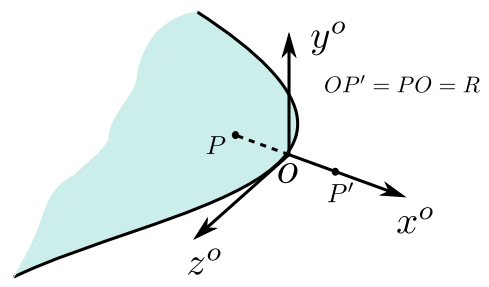

Fig. 3. Crack tip coordinate system.

VCE relies on the construction of a structured mesh along the crack front, which decreases mesh independence. However, it should be noted that in the same context of VCE, the variational form of the strain energy which involves the energy release rate and the crack extension has been developed for automatic crack growth [102,103]. The crack extension is given physical interpretation. The variational form minimizes the strain energy in a global sense and has recently been investigated in the framework of XFEM [104].

The virtual crack closure integral (VCCI) method, based on the virtual crack extension, is another alternative to extract SIFs in linear elastic fracture. Due to its simplicity and accuracy, the VCCI has been widely used in FEM and BEM [105]. While it should be noted that this method requires the structured mesh near the crack front. The path-independent $J$ integral proposed by Rice [106] is an attractive method due to its robustness regarding the relative independence in discretization and integral on domain. The method was extended into many branches based on both FEM and BEM [107-111]. The contour $J$ integral is usually cast into the equivalent domain integral form in volumebased methods as stresses are discontinuous across element edges and statically admissible smoothing/recovery techniques are cumbersome. While in BEM the contour definition can be adopted directly [112]. In order to extract mixed mode SIFs, different techniques are developed. The $J_{x}$ integrals $(x=1,2,3)$, as the components of the $\mathbf{J}$ integral, can be directly used to evaluate the SIFs. However, the evaluation of $J_{2}$ and $J_{3}$ (or $G_{I I I}$ ) exhibits numerical difficulties due to the singularity [113]. The $J_{1}$ integral (or $J$ integral) can also be used to extract mixed mode SIFs, with some auxiliary operation. One approach is to decompose the displacement and stress fields into symmetric and antisymmetric portions with a structured mesh along the crack front, then the three modes of the $J$ integral can be extracted directly [114-117]. The other method known as the $M$ integral (or interaction energy integral), was developed by introducing asymptotic fields as an auxiliary solution [108] has been extended in (X)FEM [118,31] and BEM [119].

In the present work, both the VCCI and the contour $M$ integral have been investigated for the calculation of SIFs in fracture analysis via the 3D isogeometric BEM. A point-wise crack tip coordinate system is established along the crack front as in Fig. 3. The physical quantities are all in the crack tip local coordinate system thus the superscript ' $o$ ' is omitted in the section.

\subsubsection{Contour $M$ integral}

The definition of the $J_{k}$ integral stems from two dimensions as:

$$
J_{k}:=\lim _{\Gamma_{\epsilon} \rightarrow 0} \int_{\Gamma_{\epsilon}}\left(W \delta_{j k}-\sigma_{i j} u_{i, k}\right) n_{j} \mathrm{~d} \Gamma=\lim _{\Gamma_{\epsilon} \rightarrow 0} \int_{\Gamma_{\epsilon}} P_{k j} n_{j} \mathrm{~d} \Gamma, \quad k=1,2
$$

where $P_{k j}$ is the Eshelby tensor, $W=1 / 2 \sigma_{i j} \epsilon_{i j}$ is the strain energy density. $\Gamma_{\epsilon}$ is a small contour with radius $R$ centered at the point ' $o$ ' on the crack front in the ' $x_{o}-y_{o}$ ' plane and $n_{j}$ is the unit outward normal of $\Gamma_{\epsilon}$.

This 2D definition can be extended to a three dimensional point-wise definition by taking a tubular surface around the crack front. When the contour $\Gamma_{\epsilon}$ is small enough, plane strain conditions are approximately satisfied. The contour definition can thus be used directly on the premise that a sufficiently small contour is assumed.

It is known that the $J$ integral $\left(J_{1}\right)$ and the SIFs are linked by the following expression

$$
J=G_{I}+G_{I I}+G_{I I I}=\frac{1-v^{2}}{E} K_{I}^{2}+\frac{1-v^{2}}{E} K_{I I}^{2}+\frac{1}{2 \mu} K_{I I I}^{2},
$$

where $G_{i}$ and $K_{i}(i=I, I I, I I I)$ are the energy release rates and SIFs for the three modes of fracture.

By applying the $J$ integral under two states, one being the real state (denoted with superscript ' 1 '), the other the auxiliary state (superscript ' 2 '), and adding them together, the mixed term $M$ can be obtained by the following 
manipulation:

$$
J^{(1+2)}=\int_{\Gamma_{\epsilon}}\left[\frac{1}{2}\left(\sigma_{i j}^{(1)}+\sigma_{i j}^{(2)}\right)\left(\epsilon_{i j}^{(1)}+\epsilon_{i j}^{(2)}\right) \delta_{1 j}-\left(\sigma_{i j}^{(1)}+\sigma_{i j}^{(2)}\right) \frac{\partial\left(u_{i}^{(1)}+u_{i}^{(2)}\right)}{\partial x_{1}}\right] n_{j} \mathrm{~d} \Gamma .
$$

Rearranging the two state terms gives

$$
J^{(1+2)}=J^{(1)}+J^{(2)}+M^{(1,2)}
$$

where

$$
\begin{aligned}
& M^{(1,2)}=\int_{\Gamma_{\epsilon}}\left[W^{(1,2)} \delta_{1 j}-\sigma_{i j}^{(1)} \frac{\partial u_{i}^{(2)}}{\partial x_{1}}-\sigma_{i j}^{(2)} \frac{\partial u_{i}^{(1)}}{\partial x_{1}}\right] n_{j} \mathrm{~d} \Gamma \\
& W^{(1,2)}=\sigma_{i j}^{(1)} \epsilon_{i j}^{(2)}=\sigma_{i j}^{(2)} \epsilon_{i j}^{(1)} .
\end{aligned}
$$

Combined with Eq. (40), the following relationship can be obtained for the $M$ integral,

$$
M^{(1,2)}=\frac{2\left(1-v^{2}\right)}{E}\left(K_{I}^{(1)} K_{I}^{(2)}+K_{I I}^{(1)} K_{I I}^{(2)}\right)+\frac{1}{\mu} K_{I I I}^{(1)} K_{I I I}^{(2)} .
$$

The SIFs can then be extracted by making specific selections for state 2 . For example, if the auxiliary mode is the pure mode $I I I$ asymptotic fields with $K_{I}^{(2)}=0, K_{I I}^{(2)}=0, K_{I I I}^{(2)}=1$ then, $K_{I I I}$ in real state 1 can be found as

$$
K_{\text {III }}^{(1)}=\mu M^{(1, \text { mode III) }}
$$

$K_{I}$ and $K_{I I}$ can be obtained in a similar fashion. In this work the first order asymptotic displacement and stress solutions (see Appendix A) are selected as auxiliary fields.

\subsubsection{Virtual crack closure integral}

In the VCCI, the strain energy release rate is equal to the work done by closing the crack along its extension. The modes of the strain energy release rate are given by

$$
\begin{aligned}
G_{I} & =\frac{1}{2 R} \int_{0}^{R} \sigma_{y y}(x) \llbracket u_{y}(R-x) \rrbracket \mathrm{d} x, \\
G_{I I} & =\frac{1}{2 R} \int_{0}^{R} \sigma_{x y}(x) \llbracket u_{x}(R-x) \rrbracket \mathrm{d} x, \\
G_{I I I} & =\frac{1}{2 R} \int_{0}^{R} \sigma_{y z}(x) \llbracket u_{z}(R-x) \rrbracket \mathrm{d} x,
\end{aligned}
$$

where $O P^{\prime}=R$ is the virtual crack advance. For the evaluation of $\llbracket u_{j}(R-x) \rrbracket$ on $P O$, the point inversion algorithm needs to be performed in order to find the parametric coordinates in the crack modeled by the NURBS surface [84]. The domains of these integrals $O P^{\prime}$ and $P O$ are discretized by a single linear element [120]. $R$ is identical for all the sample points on the crack front. Then $K_{I}, K_{I I}$ and $K_{I I I}$ can be computed according to Eq. (40).

\subsection{Paris law}

The Paris-based laws are typical empirical relation linking the increment in crack advance $\mathrm{d} a$ occurring during $\mathrm{d} N$ cycles to the SIF amplitude, though empirically obtained coefficients $C$ and $m$. The simplest expression of Paris law reads:

$$
\frac{\mathrm{d} a}{\mathrm{~d} N}=C(\Delta K)^{m}
$$

For mixed mode fracture, $K$ is taken as the equivalent SIF $K_{\text {eq }}$ which is given as [7]:

$$
K_{\mathrm{eq}}=\sqrt{K_{I}^{2}+K_{I I}^{2}+(1+v) K_{I I I}^{2}}
$$


a

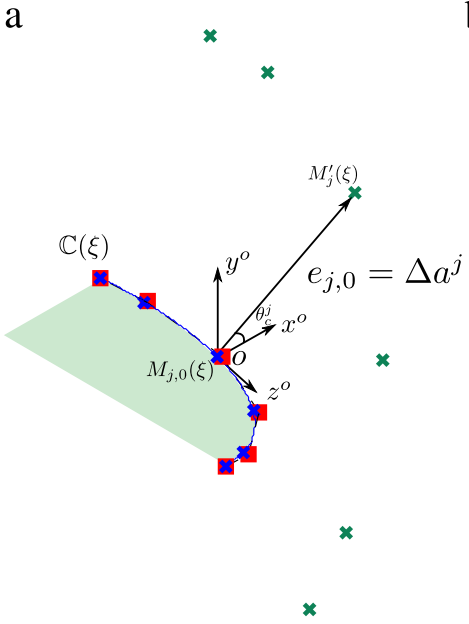

b

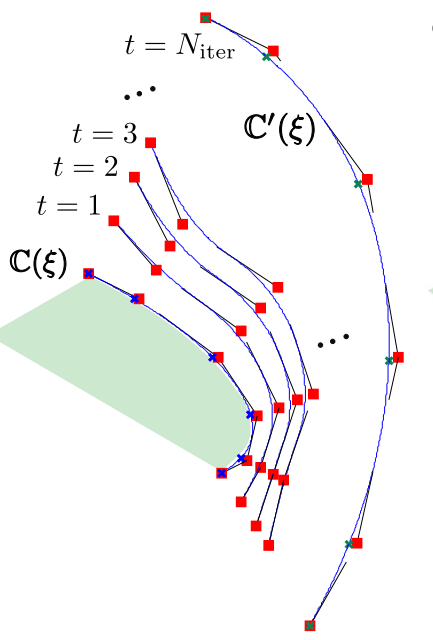

$\mathrm{c}$

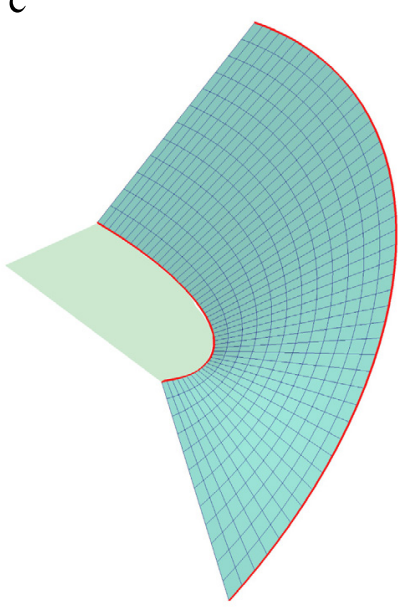

Fig. 4. Crack surface updating for a growth step. The red rectangles denote the control points of NURBS curves. (a) The crack front curve $\mathbb{C}(\xi)$ is extracted from the original crack surface. The Greville Abscissae is used to obtain sample points (blue crosses). New position of those sample points (green crosses) $M_{j}^{\prime}(\xi)$ is determined by $\Delta a^{j}$ and $\theta_{c}^{j}$ from the fracture law. (b) New front curve $\mathbb{C}^{\prime}(\xi)$ is obtained by moving the control points of $\mathbb{C}(\xi)$ iteratively until the criterion in the algorithm is met. (c) A new crack surface is obtained by linear interpolation between the two curves $\mathbb{C}(\xi)$ and $\mathbb{C}^{\prime}(\xi)$. (For interpretation of the references to color in this figure legend, the reader is referred to the web version of this article.)

It should be noted that the crack propagation velocity could be varied for the points along the front. In a single propagation step, the crack advance for each point is regularized by the user-specified maximum increment of crack advance $\Delta a^{\max }$,

$$
\Delta a^{i}=C\left(\Delta K_{\mathrm{eq}}^{i}\right)^{m} \frac{\Delta a^{\max }}{C\left(\Delta K_{\mathrm{eq}}^{\max }\right)}=\Delta a^{\max }\left(\frac{\Delta K_{\mathrm{eq}}^{i}}{\Delta K_{\mathrm{eq}}^{\mathrm{max}}}\right)^{m} .
$$

The maximum hoop stress criterion is used to determine the direction of crack propagation. We assume, at each point on the crack front, that the crack propagates in the direction $\theta_{c}$ such that the hoop stress is maximum. This is given by the following expression [121]

$$
\theta_{c}=2 \arctan \left[\frac{-2\left(K_{I I} / K_{I}\right)}{1+\sqrt{1+8\left(K_{I I} / K_{I}\right)^{2}}}\right]
$$

\subsection{Crack surface updating algorithm}

Crack propagation is realized geometrically by advancing the crack front so that the new crack front curve $\mathbb{C}^{\prime}(\xi)$ passes through the new positions of the sample points on the old crack front curve $\mathbb{C}(\xi)$ which is parametrized by the knot vector $\Xi=\left\{\xi_{1}, \xi_{2}, \ldots, \xi_{n+p+1}\right\}, n$ is the number of basis functions. We define the sample points on $\mathbb{C}(\xi)$ to be $M_{j}=\mathbb{C}\left(\xi_{j}\right), j=1,2, \ldots, N$, and the set of points with new position to be $M_{j}^{\prime}=\mathbb{C}^{\prime}\left(\xi_{j}\right)$ and we set $N=n$ here. Note that each $M_{j}^{\prime}$ is calculated via the fracture parameters $K$ and $\Delta a$ introduced in the previous section. We adopt the algorithm described in [96] to generate a new curve which passes through all the new sample points by updating the control points of the old curve through an iterative process. For $t$ th iteration step, we define the error vector $\mathbf{e}_{t}$ as:

$$
e_{j, t}=\overrightarrow{M_{j, t} M_{j}^{\prime}}, \quad j=1,2, \ldots, N
$$

Note that when $t=0, e_{j, t}=\overrightarrow{M_{j, 0} M_{j}^{\prime}}=\overrightarrow{M_{j} M_{j}^{\prime}}=\Delta a_{j}$ which is the crack advance of the point on the crack front. If $\left\|\mathbf{e}_{t}\right\|<t o l$, the iteration ceases and the new crack front curve is obtained (see Fig. 4).

To update the control points, we define a motion vector $\mathbf{m}_{t}$ for the control points such that at the $t$ th iteration step:

$$
P_{i, t}=P_{i, t-1}+m_{i, t}, \quad i=1,2, \ldots, n,
$$


with $P_{i, 0}=P_{i}$ which are the control points of the old crack front $\mathbb{C}$. The motion vector $\mathbf{m}_{t}$ can be computed as:

$$
m_{i, t}=\frac{1}{N} \sum_{j=1}^{N} f_{i j} e_{j, t-1}, \quad t>1,
$$

where $f_{i j}=f_{i}\left(\xi_{j}\right)$ are the influence functions corresponding to each constraint $M_{j}^{\prime}$. We choose the influence functions to be the NURBS basis functions which are used to describe the curve, i.e. $f_{i}(\xi)=R_{i}(\xi)$. The parameter coordinate $\xi_{j}$ of each $M_{j}$ should satisfy $\xi_{j} \in\left[\xi_{i}, \xi_{i+p+1}\right]$. We use the Greville Abscissae to generate the sample points to make sure the influence functions associated with each $M_{j}^{\prime}$ are linearly independent [96]. Finally, the error vector is calculated in a recursive way:

$$
e_{j, t}=e_{j, t-1}-\frac{1}{N} \sum_{k=1}^{N} \sum_{i=1}^{n} R_{i j} f_{i k} e_{k, t-1},
$$

where $R_{i j}=R_{i}\left(\xi_{j}\right)$. The details for updating the crack front is given in Algorithm 1. Once the new crack front curve is obtained, the new crack surfaces can be generated by lofting along the crack extension direction from the old curve to the new curve. The generated crack surfaces can be merged into the old crack surfaces with either a $C^{0}$ joint or a $C^{1}$ joint. In this work a $C^{0}$ merging is adopted.

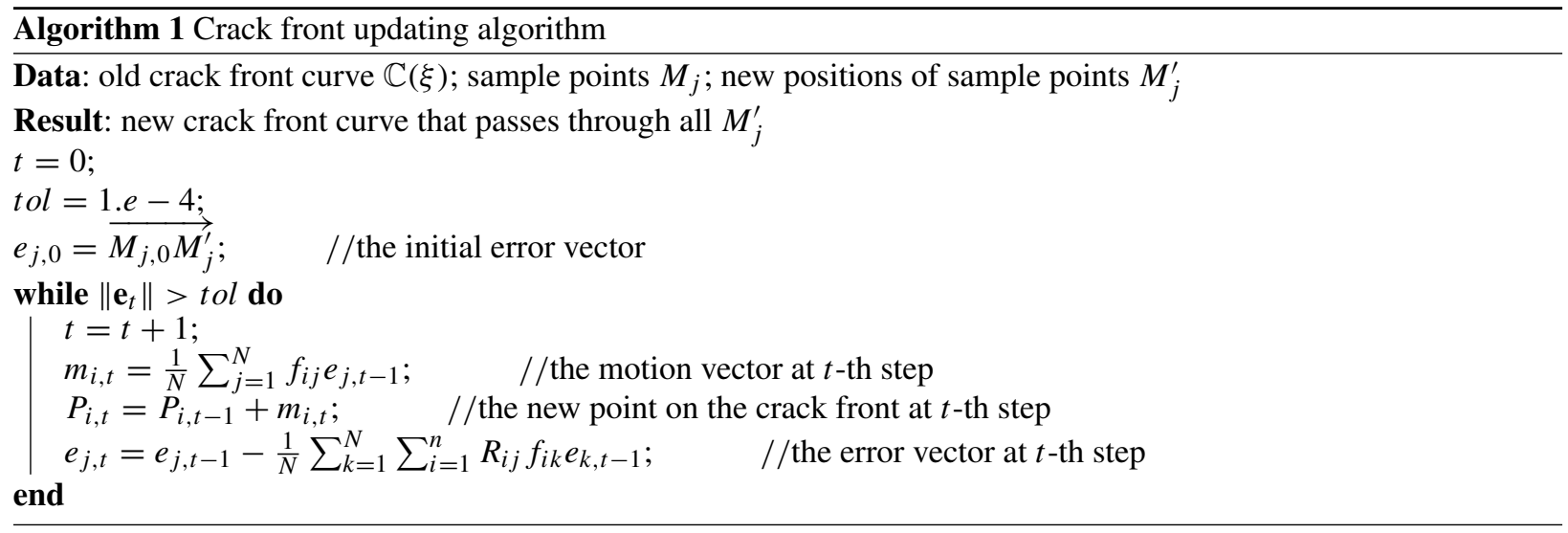

\section{Numerical examples}

In this section, numerical examples are presented as follows: In the first two examples we study penny-shaped and elliptical static cracks. We conduct (a) detailed study of suggested integration schemes; (b) convergence analysis; (c) accuracy tests for SIF calculations. In the next two examples we demonstrate the application of the crack propagation algorithm for fatigue crack growth simulation of an in-plane penny crack and inclined elliptical crack. The Young's modulus $E=1000$ and Poisson's ratio $v=0.3$ for all cases. The relative error in the $L_{2}$ norm of COD is computed as

$$
e_{L_{2}}=\sqrt{\frac{\int_{S}\left(\llbracket \mathbf{u} \rrbracket-\llbracket \mathbf{u} \rrbracket_{\text {ext }}\right)\left(\llbracket \mathbf{u} \rrbracket-\llbracket \mathbf{u} \rrbracket_{\text {ext }}\right)^{\mathrm{T}} \mathrm{d} S}{\int_{S} \llbracket \mathbf{u} \rrbracket_{\text {ext }} \llbracket \mathbf{u} \rrbracket_{\text {ext }}^{\mathrm{T}} \mathrm{d} S},},
$$

where the subscript 'ext' denotes the analytical solution of COD.

\subsection{Penny-shaped crack}

Suppose a penny-shaped crack is subjected to the remote tension $\sigma_{0}$, i.e. $\mathbf{t}^{\infty}=\left(0,0, \sigma_{0}\right)$. The radius of circle is $a$. The inclination angle is $\varphi$ and circular angle $\theta$ is defined in the crack plane $(O x y)$ as in Fig. 5. The analytical solution 


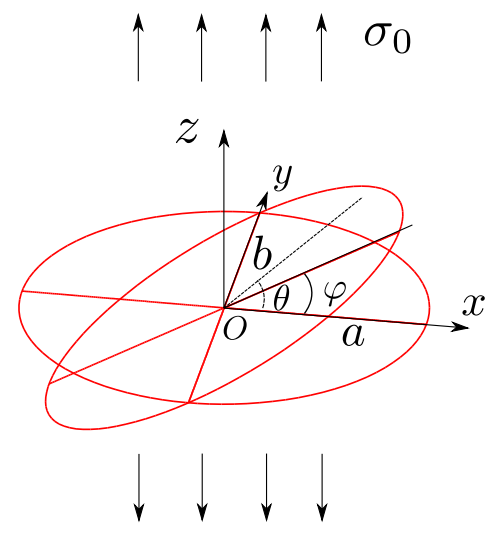

Fig. 5. Geometry for penny-shaped crack $(a=b)$ and elliptical crack $(a \neq b)$.

for the SIFs read:

$$
\begin{aligned}
K_{I}(\varphi) & =\frac{2}{\pi} \sigma_{0} \sqrt{a \pi} \cos ^{2} \varphi, \\
K_{I I}(\varphi, \theta) & =\frac{4}{\pi(2-v)} \sigma_{0} \sqrt{a \pi} \cos \varphi \sin \varphi \cos \theta, \\
K_{I I I}(\varphi, \theta) & =\frac{4(1-v)}{\pi(2-v)} \sigma_{0} \sqrt{a \pi} \cos \varphi \sin \varphi \sin \theta .
\end{aligned}
$$

In particular, when the crack plane is horizontal $(\varphi=0)$, the analytical normal displacement is given as:

$$
u_{z}(r, \theta, 0)=\frac{2(1-v) \sigma_{0}}{\pi \mu} \sqrt{a^{2}-r^{2}}, \quad r \leqslant a .
$$

\subsubsection{Singular integration test}

The problem is modeled by COD equation (10), so that a single NURBS patch is necessary to represent the crack. Fig. 6(a) illustrates the NURBS Surface which is composed of 4 elements and Fig. 6(b) is obtained by 1 time uniform mesh refinement based on (a). (c) and (d) present the knot lines in parametric space. For the sake of convenient description in the following context, we name the $\xi$ direction as the angular direction of the disc and the $\eta$ direction as the radial direction and it can be observed that $\eta=1$ represents the crack front (Fig. 6). The control points for (a) are given in Appendix B. It can be seen that the control points are coinciding at the pole of the disc. The collocation points are moved aside from the pole in order not to locate at the degenerated point. The NURBS basis functions associated with the pole, however, are enforced to be $C^{0}$ through sharing the same degrees of freedom. The BIEs from these moved collocation points are merged into one equation.

We note that the COD solution only varies in the radial direction and is constant in the angular direction, thus 4 elements are used in the angular direction. This will lead to high aspect ratio of each element with the refinement in the radial direction. Fig. 9 compares the $L_{2}$ norm error in COD for $\varphi=0$. 'ngp $s$ ' denotes the number of Gauß points in the angular direction in each sub-triangle. By 'original SST', we mean a direct use of the method and by 'improved SST', the SST with conformal and Sigmoidal transformation. It can be observed that

- when ngp $s=30$, the errors of the original SST and improved SST are comparable. However, the error from the original SST is non-uniformly distributed whilst the improved SST provides a more uniform error distribution;

- when $\operatorname{ngp} \_=18$, the error from the original SST increases significantly $\left(e_{L_{2}}=1.467716 \mathrm{e}-1\right)$, while the improved SST maintains the same accuracy as for $\operatorname{ngp} \_s=30$;

- the error is larger near the crack front. This is due to the crack tip singularity.

We conclude that the original SST requires more Gauß points for the same accuracy level as the improved SST. If we move the knot $(\eta=0.875)$ next to the crack front in the radial direction closer to the crack front $(\eta=0.94)$ (the knot indicated by red solid line is moved to red dashed line in Fig. 8) and repeat the comparison of Fig. 10, we find that 


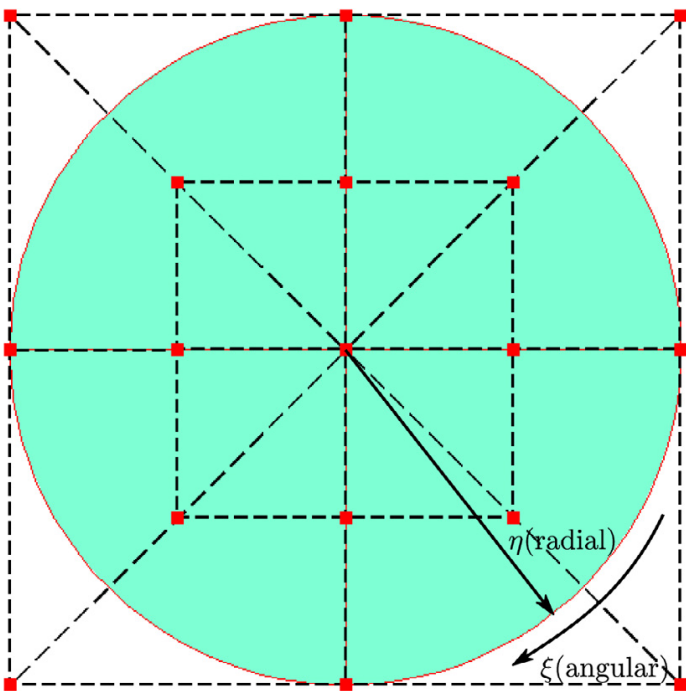

(a) Coarse mesh: the initial geometry for penny crack.

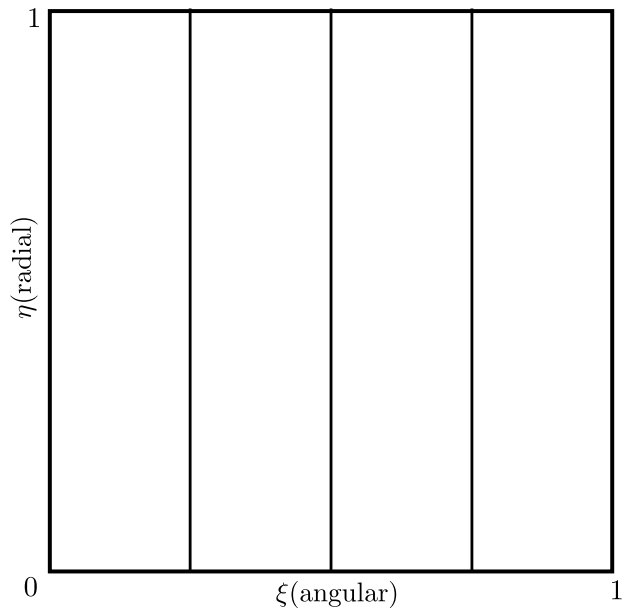

(c) Parametric space for coarse mesh.

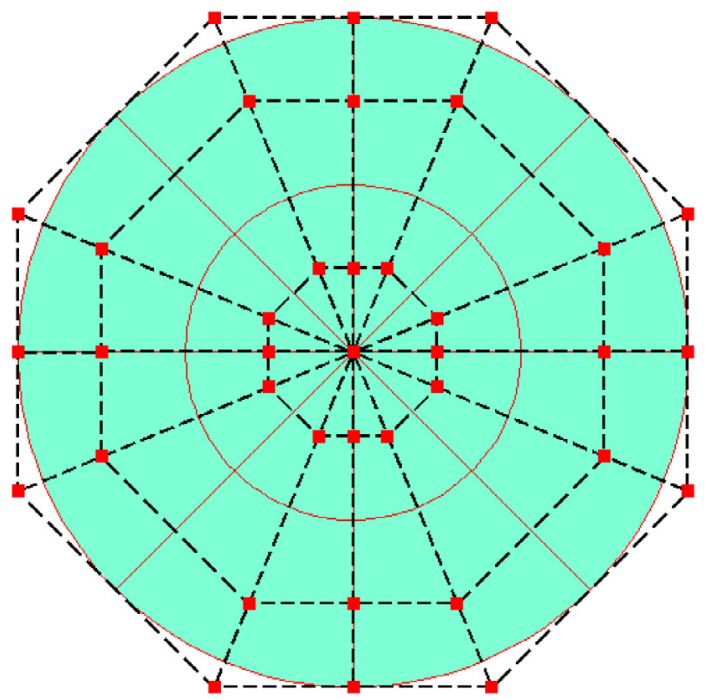

(b) Finer mesh: the 1 time uniform refinement in parameter space based on initial geometry.

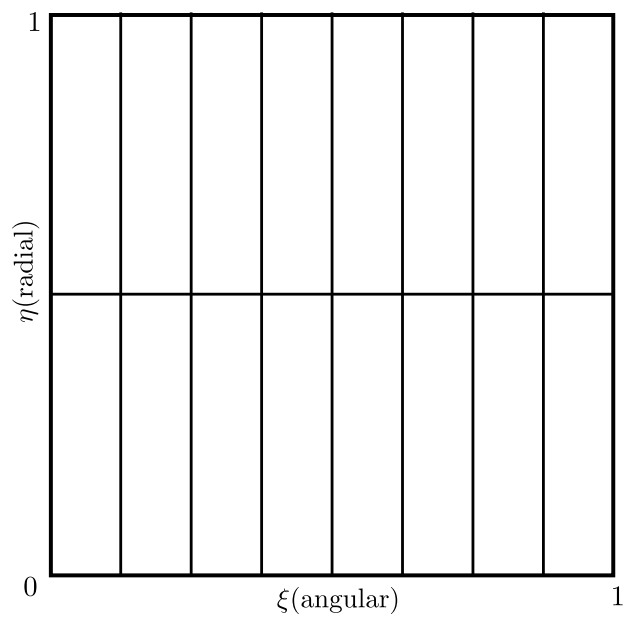

(d) Parametric space for finer mesh.

Fig. 6. NURBS represented ( $p=q=2$ ) penny crack. Knot vectors for coarse mesh, the red rectangles are control points: angular direction $\xi=[0,0,0,0.25,0.25,0.5,0.5,0.75,0.75,1,1,1]$, radial direction $\eta=[0,0,0,1,1,1]$. For finer mesh:angular direction $\xi=$ $[0,0,0,0.125,0.25,0.25,0.375,0.5,0.5,0.625,0.75,0.75,0.875,1,1,1]$, radial direction $\eta=[0,0,0,0.5,1,1,1]$ (For interpretation of the references to color in this figure legend, the reader is referred to the web version of this article.)

even for $\operatorname{ngp}_{-} s=30$, the original SST still gives error as large as for $\operatorname{ngp} s=18$ while the improved method gives an error of $e_{L_{2}}=1.755681 \mathrm{e}-2$, which is lower than what was shown in Fig. 9. We can refer that, due to the crack tip singularity, a refined mesh near the crack front should give a better accuracy in COD, but the original SST is sensitive to the element distortion and gives diverged results. The improved SST presents a robust application for this kind of mesh configuration.

\subsubsection{Convergence test}

We perform uniform mesh refinement in parametric space as shown in Fig. 6 until 5 times. We calculate the element size as $h=\sqrt{S_{e}^{\max }}$, where $S_{e}^{\max }$ denotes the maximum area of the elements. The convergence curves are plotted in Fig. 12. For IGABEM, the default $\alpha$ is 0.5 for both quadratic and cubic NURBS basis functions. We also compared discontinuous quadratic Lagrange element BEM (LBEM) [44] where the collocation points are moved inside each element in order to fulfill the continuity requirement in BIEs. The mesh for LBEM is generated from the NURBS 


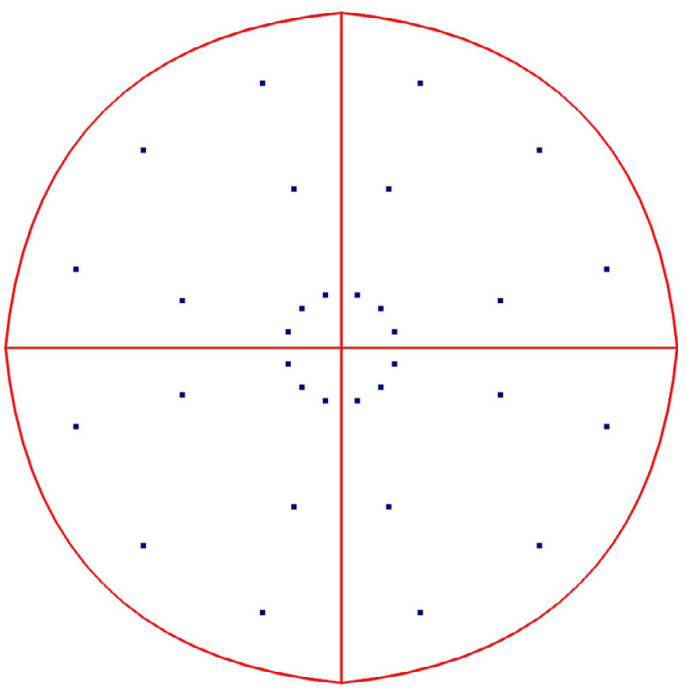

(a) Coarse mesh.

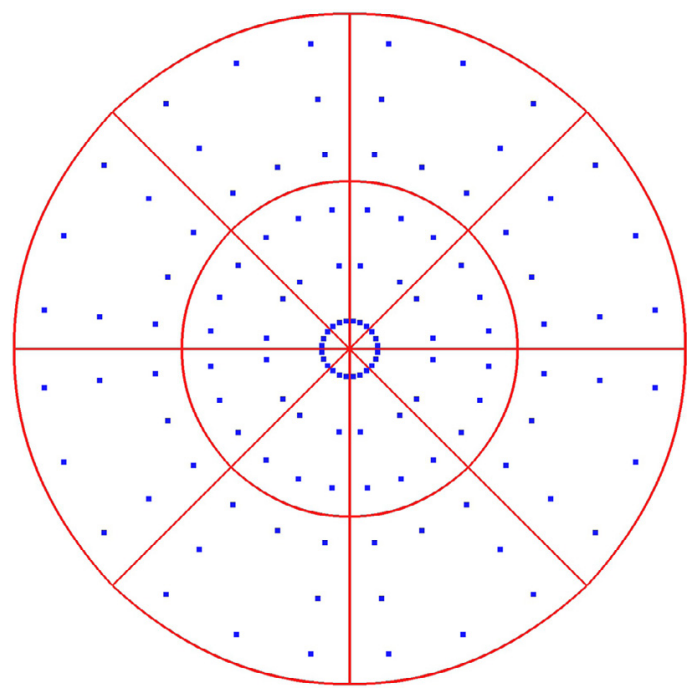

(b) Finer mesh.

Fig. 7. Discontinuous quadratic Lagrange element discretizations for penny crack. Blue dots are element nodes (collocation points) (For interpretation of the references to color in this figure legend, the reader is referred to the web version of this article.)

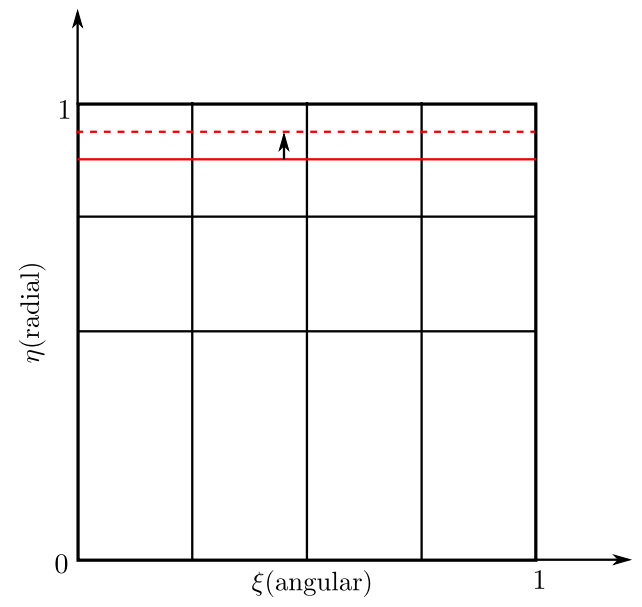

Fig. 8. Original knot vectors: angular direction $\xi=[0,0,0,0.25,0.25,0.5,0.5,0.75,0.75,1,1,1]$, radial direction $\eta=[0,0,0,0.5,0.75$, $0.875,1,1,1]$. After moving the red solid line to red dashed place, knot vectors: angular direction $\xi=[0,0,0,0.25,0.25,0.5,0.5,0.75,0.75,1$ $, 1,1]$, radial direction $\eta=[0,0,0,0.5,0.75,0.94,1,1,1]$. (For interpretation of the references to color in this figure legend, the reader is referred to the web version of this article.)

surface and then it will maintain the same topological element discretization as in IGABEM, see Fig. 7. It can be concluded that

- degree elevation of NURBS basis functions improves accuracy. Yet, the order of convergence rate (ocr) of the relative error in the $L_{2}$ norm of COD keeps almost the same value $(o c r=1)$. The deteriorated $o c r$ is due to the physical singularity along the crack front.

- When the value of $\alpha$ becomes smaller (which means the moved collocation points are closer to their original position according to Eq. (23)), the accuracy is improved. However, further deterioration in convergence rate for $\alpha=0.1$ is observed (ocr $=0.81$ ). One possible reason for this could be the near singularity is stronger in the neighbor elements when the moved collocation points get close to the element edge.

- The quadratic Lagrange basis gains an accuracy close to quadratic NURBS basis with $\alpha=0.25$. Whilst the NURBS basis with $\alpha=0.1$ outperforms the Lagrange basis in terms of accuracy. 


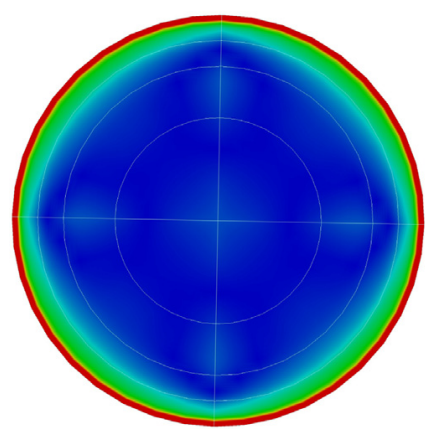

(a) Original SST, ngp $s=30, e_{L_{2}}=3.344418 \mathrm{e}-2$.

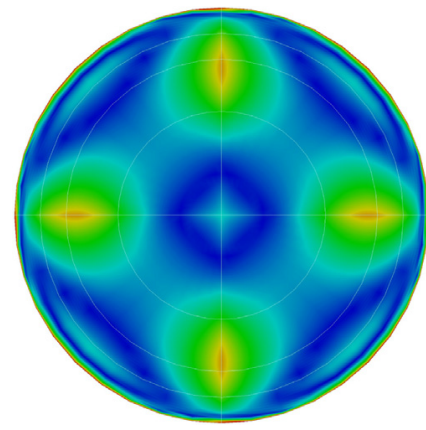

(c) Original SST, ngp_s $=18, e_{L_{2}}=1.467716 \mathrm{e}-1$.
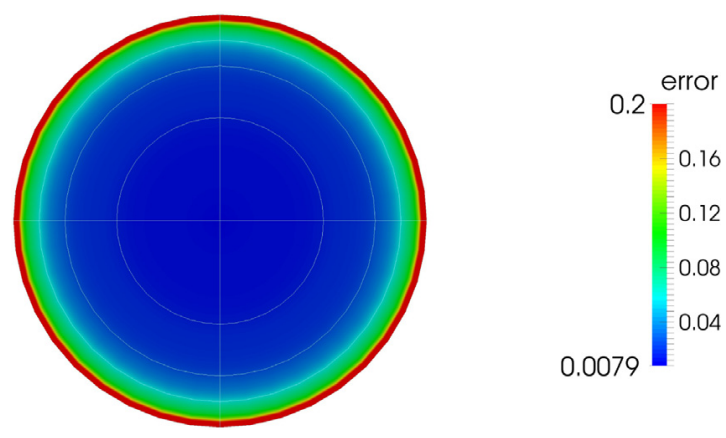

(b) Improved SST, $\operatorname{ngp} s=30, e_{L_{2}}=3.844282 \mathrm{e}-2$.
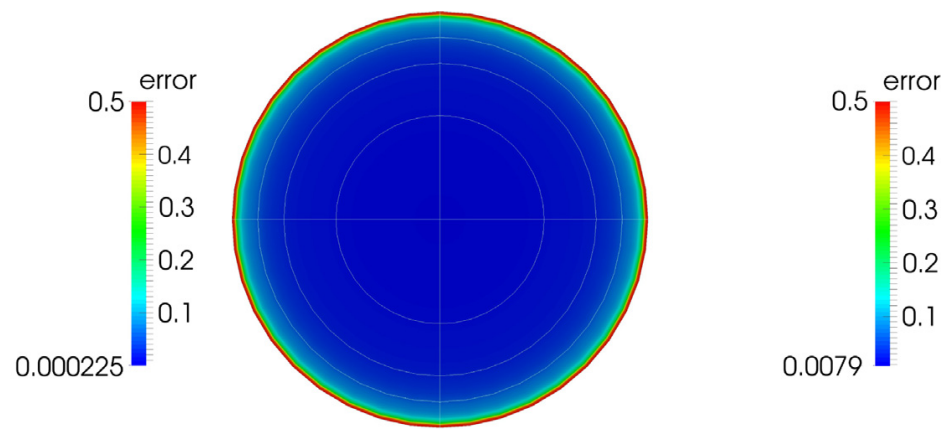

(d) Improved SST, ngp $s=18, e_{L_{2}}=3.844282 \mathrm{e}-2$.

Fig. 9. The relative error in the $L_{2}$ norm of COD for the penny crack problem for parametric space associated with red solid line. 'ngp_s' denotes the number of Gauß points in angular direction in each sub-triangle (For interpretation of the references to color in this figure legend, the reader is referred to the web version of this article.)

As is well known, uniform refinement is neither effective nor efficient to improve the accuracy for the crack problem. Thus five mesh configurations are designed, through keeping the number of elements in the angular direction while the mesh is uniformly refined by $2,4,6,8$ and 10 elements in the radial direction. The elements along the crack front is then further gradely refined by consecutive knot insertion to reduce the error caused by the crack tip singularity (Fig. 11 shows meshes 1, 3 and 5). Fig. 13 presents the result from this graded mesh refinement along with all the results from uniform refinement in parametric space in terms of degrees of freedom and all the results from IGABEM outperforms LBEM in efficiency. It can be seen that the accuracy is improved almost by one order compared to uniform refinement and the final estimate convergence rate is two times higher than for uniform refinement. This indicates the effectivity of IGABEM for fracture simulation.

\subsubsection{Stress intensity factor test}

In this subsection, the computation of SIFs is verified. Although, one of the advantages of the boundary element method for infinite domains is the necessity to discretize only the inner boundary (crack surface), the industrial applications are more concerned with problems in finite domains. Thus, instead of using the COD equation to model the penny-shaped crack in an infinite domain, we embed two overlapping crack surfaces in a cube with size $L=200 a$ such that we can compare the numerical SIFs with the analytical solution for infinite domain. Dual equations are used for this case.

Fig. 14 shows the path independence of the $M$ integral and VCCI for mode $I$ penny-shaped crack. Here ' $R$ ' denotes the virtual crack advance in VCCI and the radius of the contour in $M$ integral. It can be seen that when $R / a$ is from 0.02 to 0.08 , both methods show path dependent behavior. For $M$ integral, the error varies within $2 \%$. When the radius of contour is small, $K_{I}$ converges to analytical value; while increasing $R$, since the stress field for the crack tip is influence by other tips in the crack front, plane strain condition is not satisfied properly, the method becomes inaccurate. For VCCI, the error varies within $6 \%$ and generally a small virtual crack advance is needed. However, if $R$ is too small, difficulty in numerical evaluation of stress and COD close to crack front will arise which lead to the 


\section{ARTICLE IN PRESS}

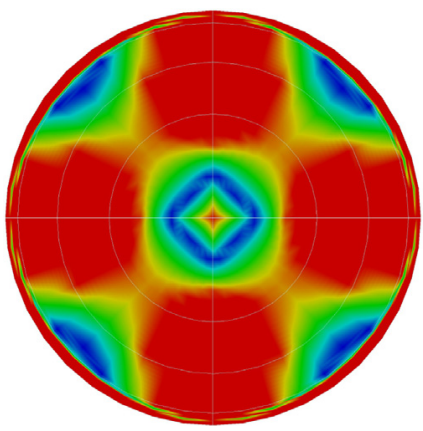

(a) Original SST, ngp $s=30, e_{L_{2}}=8.138911 \mathrm{e}-1$.

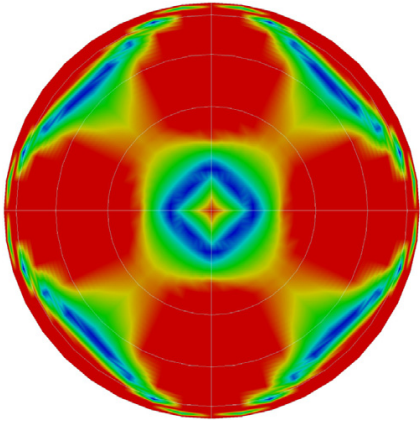

(c) Original SST, ngp_s $=18, e_{L_{2}}=7.110011 \mathrm{e}-1$.

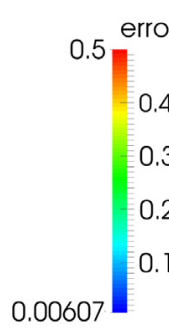

(b) Improved SST, ngp $s=30, e_{L_{2}}=1.755681 \mathrm{e}-2$

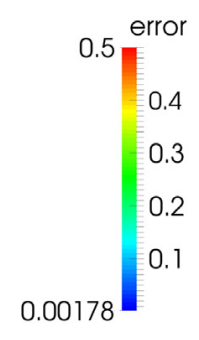

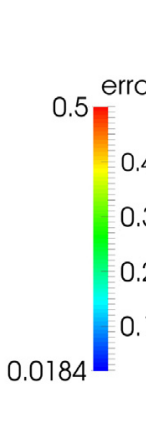

Fig. 10. The relative error in the $L_{2}$ norm of COD for the penny crack problem for parametric space associated with red dashed line (For interpretation of the references to color in this figure legend, the reader is referred to the web version of this article.)

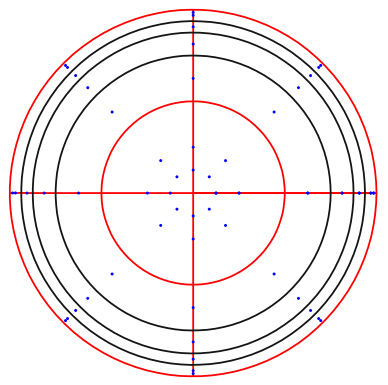

mesh 1

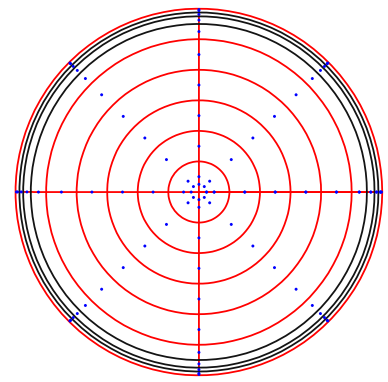

mesh 3

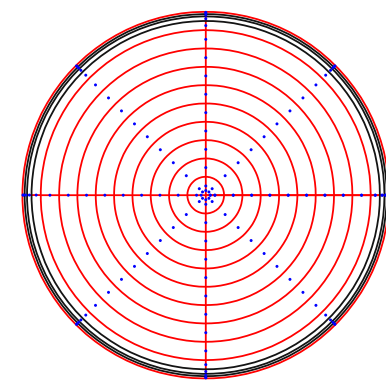

mesh 5

Fig. 11. NURBS ( $p=q=2$ ) represented crack surface meshes with 2, 6, and 10 uniform refinement in the radial direction, followed by graded refinement (with black edges) close to crack front. The blue dots are collocation points.

inaccuracy of $K_{I}$. From the figure we can also refer that $M$ integral presents a smaller reduction in error than VCCI. Therefore the $M$ integral is recommended to use for crack growth simulation by IGABEM.

Fig. 15 compares the SIFs obtained from $M$ integral with $R=0.02 a$ and VCCI with $R=0.04 a$ for the mixed mode penny-shaped crack with inclination angle $\varphi=\pi / 6$. It is seen that both methods agree well with the analytical solution. $K_{I I I}$ from $M$ integral shows deviation near $\theta=\pi / 2$ and $3 \pi / 2$. Table 1 presents the error at $\theta=0, \pi / 4$ and $\pi / 2$. It can be observed that the error of $K_{I}$ and $K_{I I}$ is within $1 \%$ by both methods, while within $7 \%$ for $K_{I I I}$ by $M$ integral. We can conclude that the IGABEM can provide accurate SIFs, and the numerical SIFs along crack front is quite smooth, although with only 4 elements in angular direction and without any smoothness operation. This gives the premise for a stable evolution for the crack growth simulation.

An inclined penny crack $(\varphi=\pi / 4)$ centered at a cylindrical bar is analyzed by IGABEM, the geometric size and boundary condition can be found in the work by Mi and Aliabadi [44]. In that work, the discontinuous Lagrange 


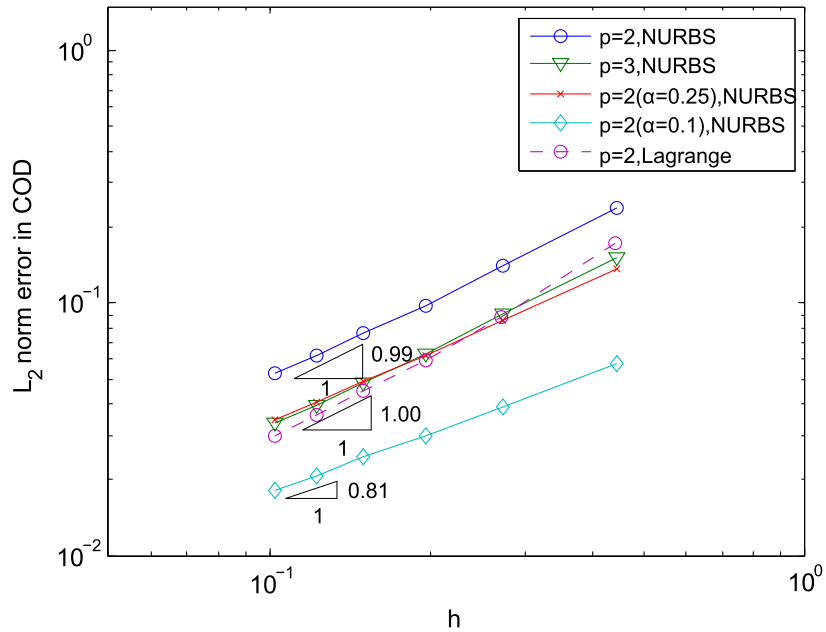

Fig. 12. The relative error in the $L_{2}$ norm of COD for penny-shaped crack. The default offset $\alpha=0.5$.

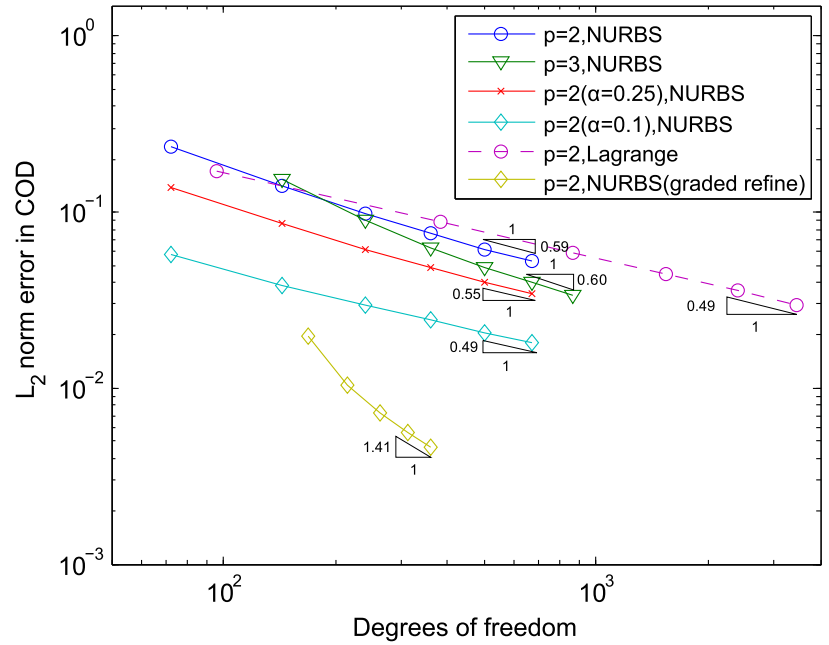

Fig. 13. The relative error in the $L_{2}$ norm of COD for penny-shaped crack. The default offset $\alpha=0.5$.

Table 1

Error of SIFs for penny-shaped crack with $\varphi=\pi / 6$.

\begin{tabular}{|c|c|c|c|c|c|c|}
\hline & $K_{I}$ & & $K_{I I}$ & & $K_{I I I}$ & \\
\hline & $\overline{\mathrm{VCCI}}$ & $M$ integral & $\overline{\mathrm{VCCI}}$ & $M$ integral & VCCI & $M$ integral \\
\hline$\theta=0$ & $2.874 \mathrm{e}-3$ & $4.311 \mathrm{e}-3$ & $7.133 e-3$ & $2.008 \mathrm{e}-3$ & $2.898 \mathrm{e}-8$ & $5.221 \mathrm{e}-9$ \\
\hline$\theta=\pi / 4$ & $2.874 \mathrm{e}-3$ & $4.311 \mathrm{e}-3$ & $7.167 \mathrm{e}-3$ & $1.983 \mathrm{e}-3$ & $1.591 \mathrm{e}-4$ & $6.243 \mathrm{e}-2$ \\
\hline$\theta=\pi / 2$ & $2.874 \mathrm{e}-3$ & $4.311 \mathrm{e}-3$ & $1.622 \mathrm{e}-8$ & $1.228 \mathrm{e}-8$ & $2.010 \mathrm{e}-4$ & $1.894 \mathrm{e}-2$ \\
\hline
\end{tabular}

elements are used to discretize the crack surfaces. Fig. 16 compares the SIFs by IGABEM and Lagrange BEM from [44]. It should be noted that for the presented result by Lagrange basis in Fig. 16, 8 elements are used in the angular direction while for IGABEM, mesh 1 in Fig. 11 (4 elements in angular direction) is used. It can be observed that the discontinuous Lagrange BEM shows fluctuation in SIFs. While the SIFs by IGABEM is smoother. Further mesh refinement needs to be performed such that the crack front can be described more accurately in order to reduce the oscillation for Lagrange BEM [44]. While in IGABEM, the crack shape is exactly represented and the local crack 


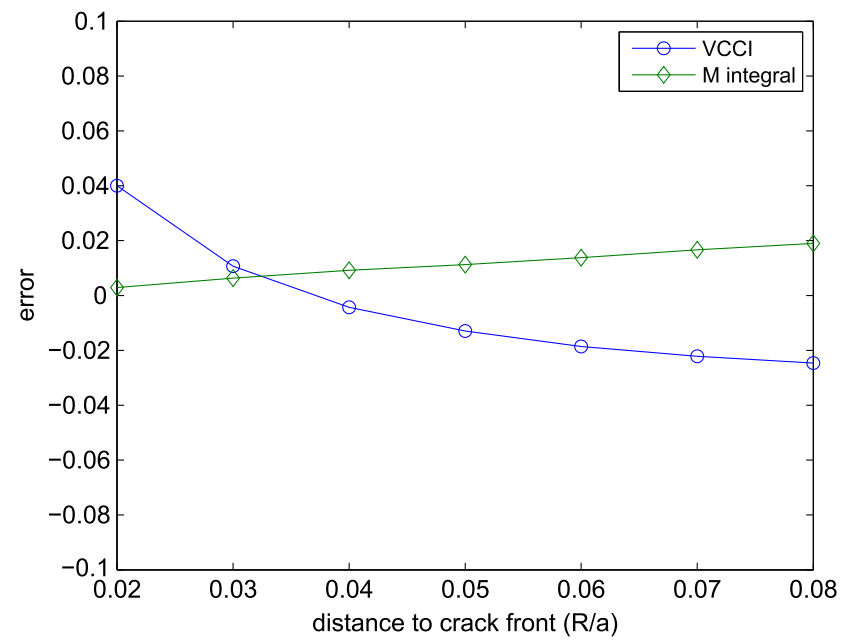

Fig. 14. Path independence verification for VCCI and $M$ integral. Here ' $R$ ' denotes the virtual crack advance in VCCI and the radius of the contour in $M$ integral.

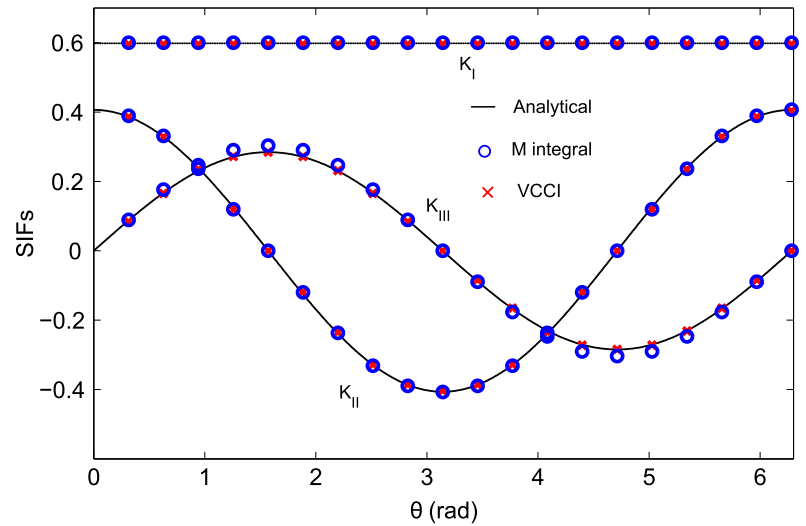

Fig. 15. Stress intensity factors for penny crack with $\varphi=\pi / 6$.

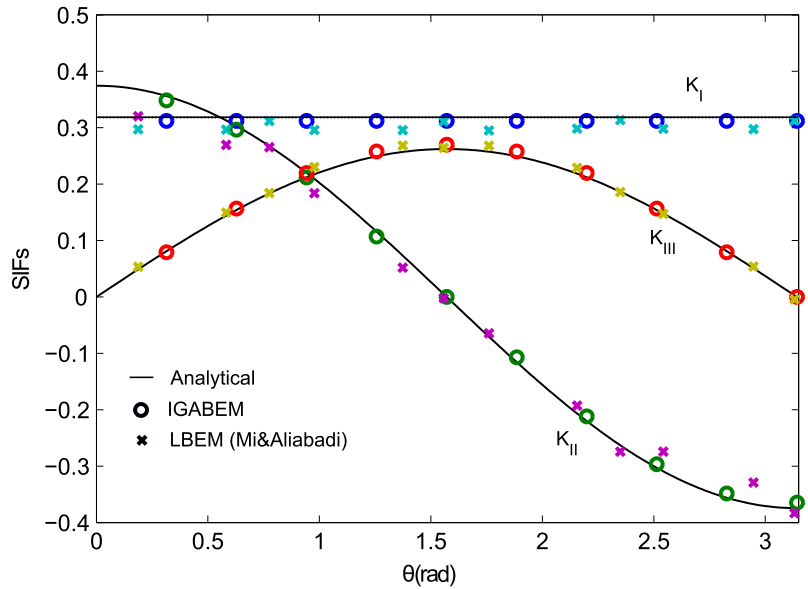

Fig. 16. Stress intensity factors for penny crack with $\varphi=\pi / 4$. LBEM denotes the discontinuous Lagrange basis BEM.

tip coordinate system is well defined thanks to the smoothness in geometry. This will lead to more accurate evaluation in fracture parameters with less elements. This example illustrates the efficiency of IGABEM for fracture simulation. 

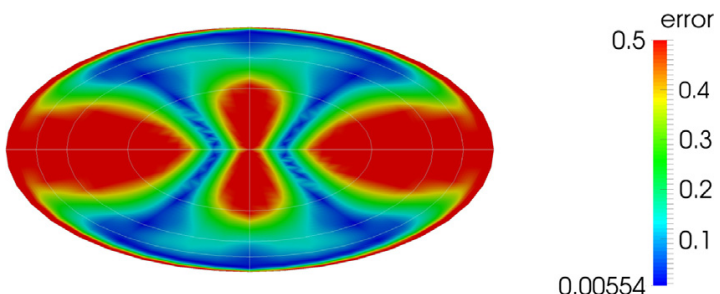

(a) Original SST, ngp_s $=18, e_{L_{2}}=4.603473 \mathrm{e}-1$.
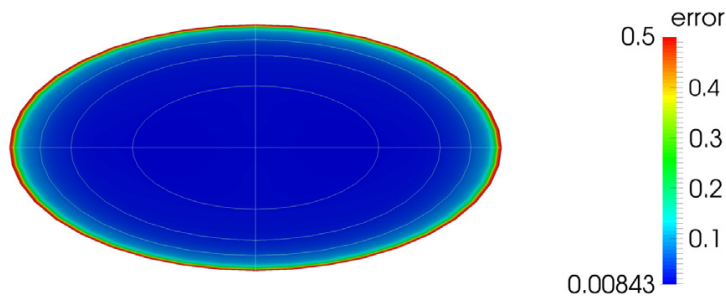

(b) Improved SST, ngp $s=18, e_{L_{2}}=3.798002 \mathrm{e}-2$.

Fig. 17. Error in crack opening displacement for elliptical crack. Knot vectors: angular direction $\xi=[0,0,0,0.25,0.25,0.5,0.5,0.75,0.75$, $1,1,1]$, radial direction $\boldsymbol{\eta}=[0,0,0,0.5,0.75,0.875,1,1,1]$

\subsection{Elliptical crack}

Suppose an elliptical crack is subjected to the remote tensile loading $\sigma_{0}$ in the normal direction, i.e. $\mathbf{t}^{\infty}=\left(0,0, \sigma_{0}\right)$. The semi-major axis is $a$, semi-minor axis $b$. The initial NURBS data can be found in Appendix B. The inclination angle is $\varphi$ and the elliptical angle $\theta$ is defined in the crack plane as in Fig. 5. The analytical SIFs read:

$$
\begin{aligned}
K_{I}(\varphi, \theta) & =\frac{\sigma_{0}}{2}(1+\cos 2 \varphi) \frac{\sqrt{b \pi} f(\theta)}{E(k)}, \\
K_{I I}(\varphi, \theta) & =\frac{\sigma_{0}}{2} \sin 2 \varphi \frac{\sqrt{b \pi} k^{2}(b / a) \cos \theta}{f(\theta) B(k)}, \\
K_{I I I}(\varphi, \theta) & =\frac{\sigma_{0}}{2} \sin 2 \varphi \frac{\sqrt{b \pi} k^{2}(1-v) \sin \theta}{f(\theta) B(k)}, \\
k^{2} & =1-\frac{b^{2}}{a^{2}}, \\
f(\theta) & =\left(\sin ^{2} \theta+\frac{b^{2}}{a^{2}} \cos ^{2} \theta\right)^{1 / 4}, \\
B(k) & =\left(k^{2}-v\right) E(k)+v \frac{b^{2}}{a^{2}} K(k),
\end{aligned}
$$

where $K(k)$ and $E(k)$ are elliptic integrals of the first kind and second kind, respectively:

$$
\begin{aligned}
& K(k)=\int_{0}^{\pi / 2} \frac{1}{\sqrt{1-k^{2} \sin ^{2} \theta}} \mathrm{d} \theta, \\
& E(k)=\int_{0}^{\pi / 2} \sqrt{1-k^{2} \sin ^{2} \theta} \mathrm{d} \theta .
\end{aligned}
$$

In particular, when $\varphi=0$, the displacement in the normal direction to the crack reads:

$$
u_{z}(x, y, 0)=\frac{2(1-v) \sigma_{0}}{\mu} \frac{b}{E(k)} \sqrt{1-\frac{x^{2}}{a^{2}}-\frac{y^{2}}{b^{2}}} .
$$

The difference of the elliptical crack and penny crack is that the mode I SIF is not a constant, due to the variation of the curvature along the crack front. The problem is modeled by COD equation (10) and mesh configuration and collocation strategy is analogous to the case of the penny-shaped crack. For elliptical cracks, the elements have high aspect ratios as well as non-orthogonal basis vectors. Fig. 17 shows that original SST presents erroneous result with $18 \mathrm{Gau} ß$ points in angular direction. While the improved SST gives a reasonable COD and error distribution.

For the convergence study, we first give the result of uniform refinement in parametric space in Fig. 19 as well as the result from LBEM with discontinuous Lagrange basis. Then the same graded mesh configurations for elliptical crack are generated as done for penny crack as in Fig. 18. Fig. 20 compares the result between uniform mesh and graded 


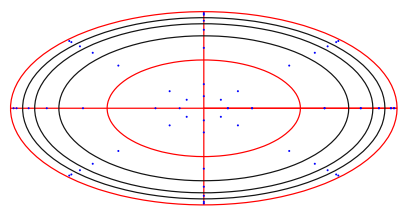

mesh 1

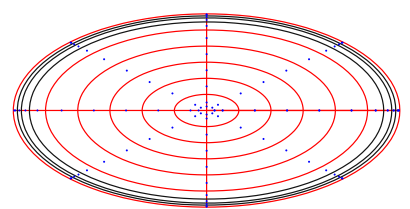

mesh 3

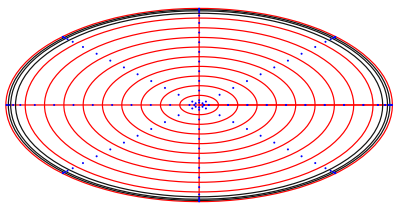

mesh 5

Fig. 18. The NURBS ( $p=q=2$ ) represented crack surface meshes with 1,5 , and 9 uniformed refinement in radial direction, followed by graded refined elements (with black edges) close to crack front. The blue dots are collocation points.

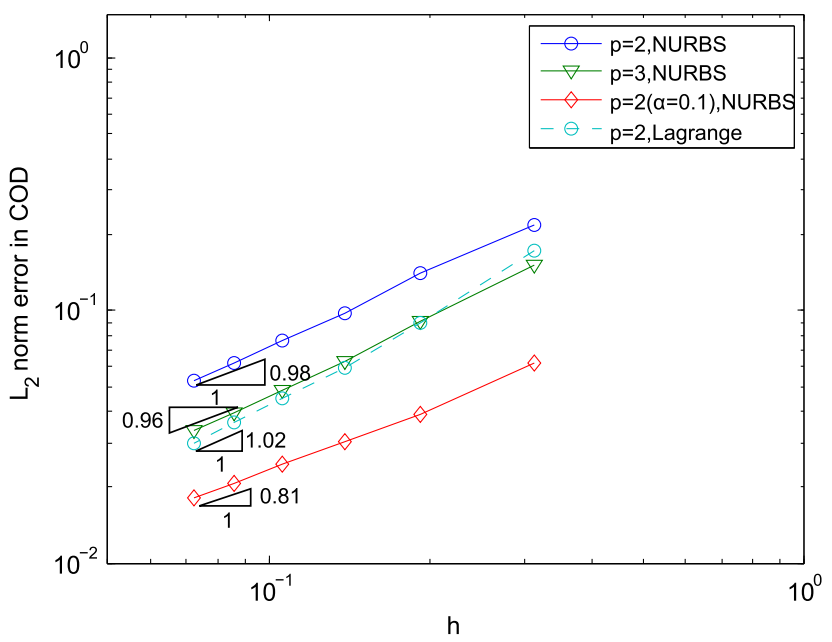

Fig. 19. $L_{2}$ norm error of COD for elliptical crack.

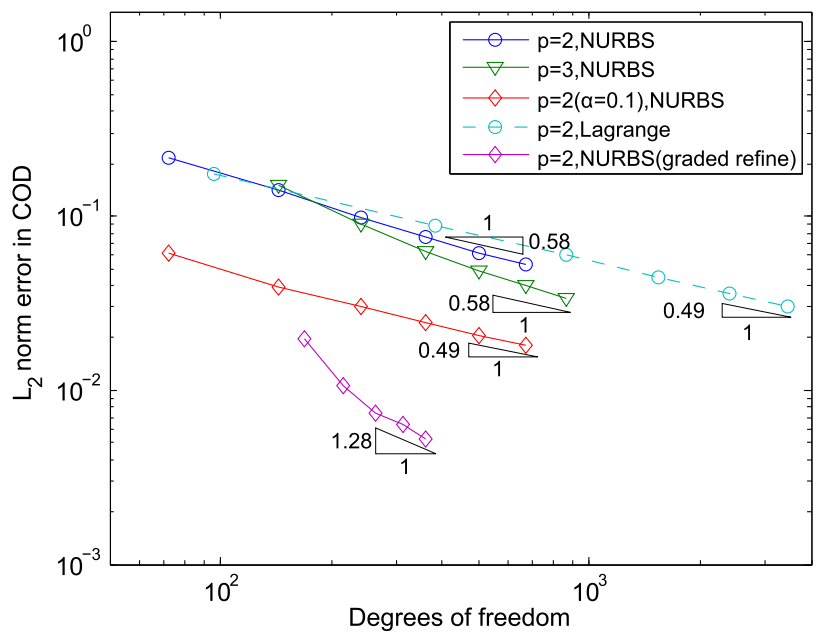

Fig. 20. $L_{2}$ norm error of COD for elliptical crack.

mesh. The convergence feature is almost the same as that of penny crack. And we can conclude that the IGABEM also suits well for modeling elliptical crack.

For the test of SIFs computation, we put two overlapping crack surfaces in a cube with size $L=200 a$ such that we could compare the numerical SIFs with the analytical solution for infinite domain. Dual equations are used. Fig. 21 compares the SIFs obtained from $M$ integral with $R=0.02 b$ and VCCI with $R=0.02 b$ for the mixed mode elliptical crack with inclination angle $\varphi=\pi / 6$. Table 2 presents the error at $\theta=0$ and $\pi / 2$ for the SIF in three modes. It can be seen that the error for all the SIFs is within $7 \%$. And the SIFs along the crack front is smooth. We note that the SIFs 


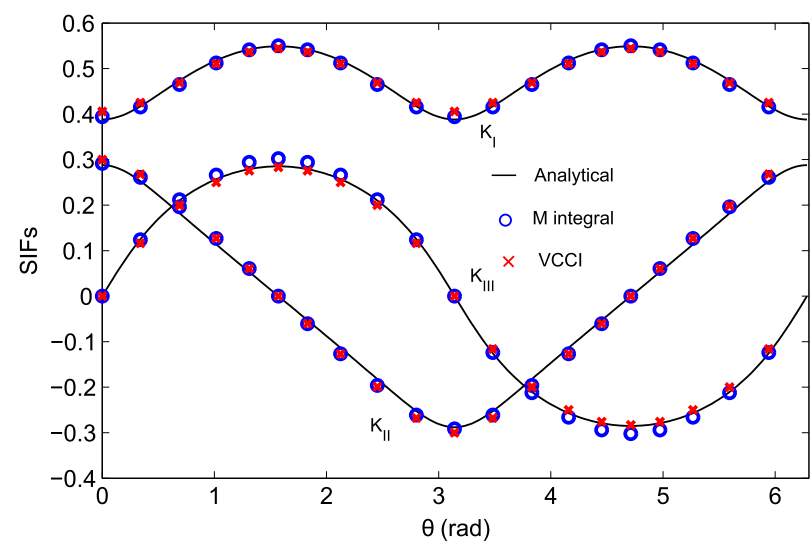

Fig. 21. Stress intensity factors for elliptical crack with $\varphi=\pi / 6$.

Table 2

Relative error of SIFs for elliptical crack with $\varphi=\pi / 6$.

\begin{tabular}{|c|c|c|c|c|c|c|}
\hline \multirow{2}{*}{ 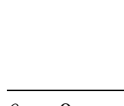 } & \multicolumn{2}{|l|}{$K_{I}$} & \multicolumn{2}{|l|}{$K_{I I}$} & \multicolumn{2}{|l|}{$K_{I I I}$} \\
\hline & $\overline{\mathrm{VCCI}}$ & $M$ integral & $\overline{\mathrm{VCCI}}$ & $M$ integral & VCCI & $M$ integral \\
\hline$\theta=0$ & $4.564 \mathrm{e}-2$ & $1.534 \mathrm{e}-2$ & $4.138 \mathrm{e}-2$ & $1.279 \mathrm{e}-2$ & $1.226 \mathrm{e}-7$ & $2.174 \mathrm{e}-7$ \\
\hline$\theta=\pi / 2$ & $8.284 \mathrm{e}-3$ & $2.214 \mathrm{e}-2$ & $6.936 \mathrm{e}-8$ & $5.152 \mathrm{e}-8$ & $6.882 \mathrm{e}-3$ & $5.959 \mathrm{e}-2$ \\
\hline
\end{tabular}

accuracy for the elliptical crack is worse than for the penny crack, which is due to the variation of the crack curvature along the crack front. Since a fixed value of $R$ is used, the singularity at the sample points near the semi-major and semi-minor axes would be different, which leads to inaccuracies in the SIFs evaluation [122]. More suitable way to estimate the SIFs for elliptical crack would be one of the future work.

\subsection{Fatigue crack growth}

\subsubsection{In-plane penny crack growth}

In this section, the crack surface updating algorithm is tested using the Paris law as a crack growth law. We first check the crack growth of the horizontal penny crack under uniform tension from Section 6.1.3. The fatigue parameters $m=2.1$ and the specified $\Delta a_{\max }=0.2 a$. We propagate 10 steps and make a comparison with the exact result and the result from the XFEM + level set method [123] (Fig. 22(a) and (b)). It can be observed that the crack front for each step agrees well with exact solution by IGABEM, while the crack front deviates gradually from the exact solution with XFEM + level set method, due to the fact that the level set method is restricted in describing the crack front exactly and this inaccuracy accumulates at each step. We then compute the crack propagation for $m=5$, and the result is presented in Fig. 22(c). We find that the numerical crack front still agrees well with the exact front, although the high index amplifies the error in crack growth rate. In order to quantitatively scale the error, we define the relative error of the numerical crack front $\Gamma_{\text {num }}$ to the exact front $\Gamma_{\text {ext }}$ as:

$$
\begin{aligned}
E_{f}(\mathbf{x}) & =\frac{\left|\Gamma_{\text {num }}(\mathbf{x})-\Gamma_{\text {ext }}(\mathbf{x})\right|}{\Delta a}, \\
\text { error } & =\frac{\int_{\Gamma} E_{f}(\mathbf{x}) \mathrm{d} \Gamma_{\text {ext }}(\mathbf{x})}{\int_{\Gamma} \mathrm{d} \Gamma_{\text {ext }}(\mathbf{x})} .
\end{aligned}
$$

Fig. 23 gives the relative error of the numerical crack front by IGABEM. It can be seen that the error accumulates in a slow speed and the difference of $m=5$ and $m=2.1$ is small, although the error for $m=5$ is larger than $m=2.1$ as expected. This test shows the proposed crack propagation scheme has the ability to grow cracks in a stable and accurate manner, thanks to the smoothness in the stress field and SIFs solution and to the exact representation of the geometry of the crack during the crack growth. 


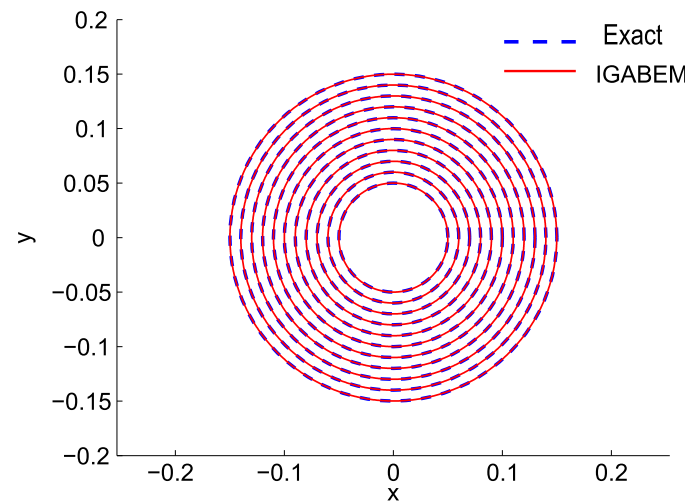

(a) IGABEM, $m=2.1$.

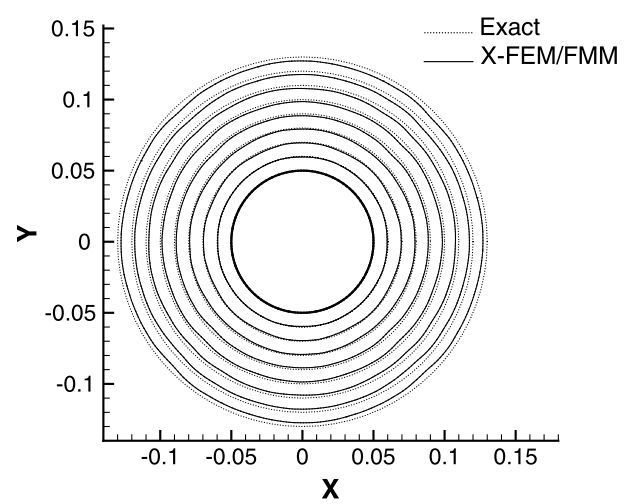

(b) XFEM/FMM, $m=2.1$, Sukumar et al. 2003.

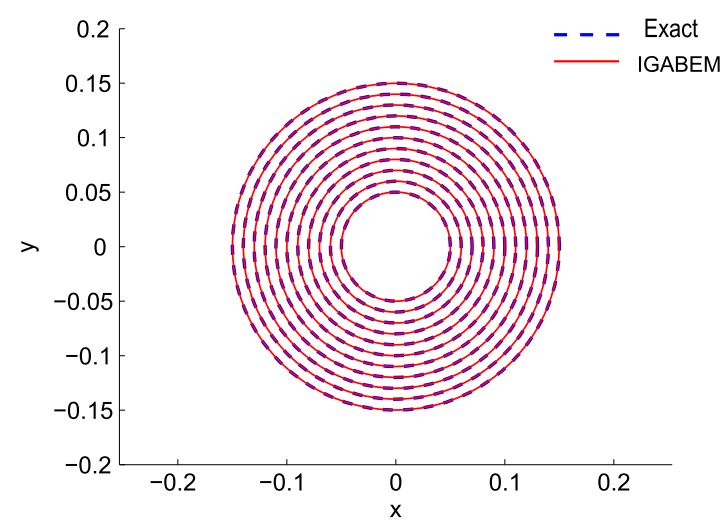

(c) IGABEM, $m=5$.

Fig. 22. Fatigue crack growth of the first 10 steps of a penny crack.

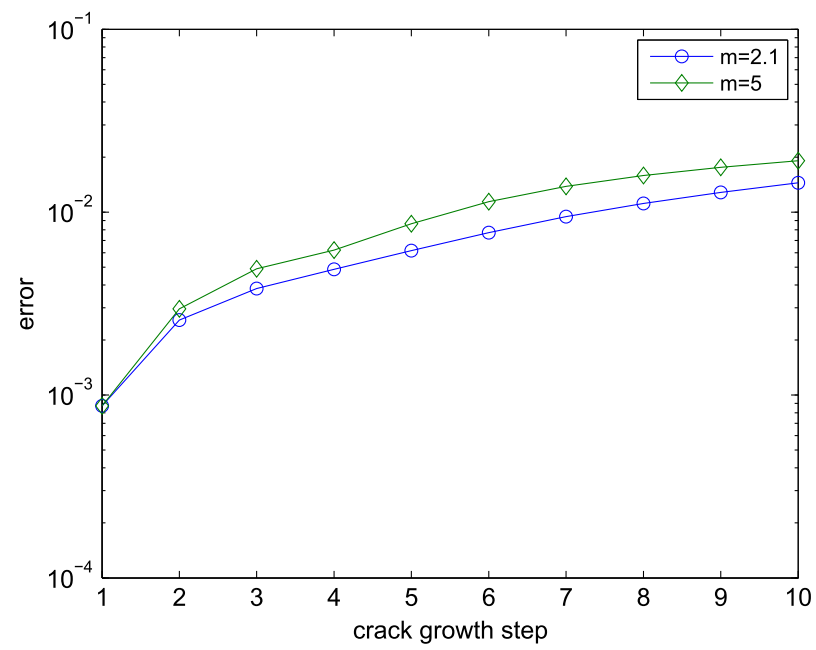

Fig. 23. Relative error of the crack front (Eq. (61)) for each crack growth step by IGABEM.

\subsubsection{Inclined elliptical crack growth}

Finally, we simulate crack growth for an elliptical crack with inclination angle $\varphi=\pi / 6$ modeled by the dual equations in a finite domain taken from Section 6.2. Fig. 24 illustrates the 2nd, 5th and 10th of the propagation step. 


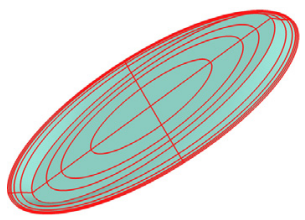

(a) Step 2 .

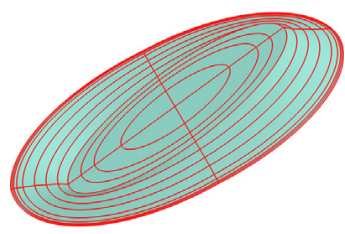

(b) Step 5 .

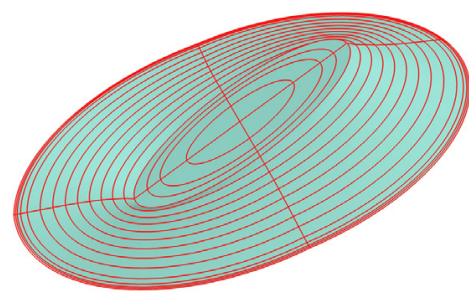

(c) Step 10 .

Fig. 24. Fatigue crack growth simulation of an elliptical crack.

\section{Conclusions}

Starting from computer aided design (CAD) NURBS data, as input, we formulated, implemented and described in detail a numerical method to simulate crack propagation in 3D, directly from the NURBS representation of the domain and which requires no mesh generation or regeneration as the cracks propagate. We investigated the advantages and drawbacks of the approach, and proposed a number of improvements to the naïve approach which makes the method amenable to realistic 3D damage tolerance assessment problems encountered in industry.

The key idea is to use the same NURBS basis functions have been used for the discretization of the geometry and crack and for the approximation of the displacement and traction fields. We used the singularity subtraction technique (SST) proposed in [83] for the treatment of (hyper-)singular integrals in the boundary element method. We extended the improved SST [87] to quadrilateral elements such that it can be applied to tensor-product NURBS basis functions. We used both the crack opening displacement (COD) form and the dual equations of IGABEM to model the crack. We tested two methods to extract SIFs: the contour-based $M$ integral and the virtual crack closure approach. We also proposed and verified an algorithm to propagate the NURBS-represented crack surface. The conclusions and highlights of this work include:

- The proposed singular integration scheme preserves the quadratic accuracy for highly distorted elements which commonly arise in isogeometric analysis. This enables a robust implementation of IGABEM;

- Through graded mesh refinement in the direction where the crack tip singularity varies, the convergence rate can be improved by a factor of 2 and the accuracy improved by one order, compared to uniform refinement. This shows the promise of IGABEM for fracture problem;

- A local crack tip system is setup naturally and uniquely thanks to the NURBS representation of the crack surface. Combining this approach with the high-order continuity in stress provided by the NURBS-based BEM, the SIFs computed along the crack front are smooth and accurate;

- The proposed algorithm for crack propagation is stable, even for high exponent values in the Paris law, due to the smoothness in the crack front geometry and the accuracy of the SIFs.

The use of CAD geometry directly for fracture simulation provides benefits. Nevertheless, specific difficulties to model surface cracks must be overcome. Introducing and growing these edge-discontinuities into a NURBS patch at an arbitrary position requires particular care, due to the higher order continuity of the basis functions. Future work will focus on the propagation of surface cracks. Dealing with multi-patch geometries and handling crack propagation through patch interfaces is another important avenue of investigation to extend and complement the method proposed here. Meanwhile, the fast solution as proposed in [42] is being implemented to accelerate the simulations.

\section{Acknowledgments}

The first and last authors would like to acknowledge the financial support of the Framework Programme 7 Initial Training Network Funding under grant number 289361 'Integrating Numerical Simulation and Geometric Design Technology'. S.P.A. Bordas also thanks partial funding for his time provided by the UK Engineering and Physical Science Research Council (EPSRC) under grant EP/G069352/1 Advanced discretization strategies for 'atomistic' nano CMOS simulation; the EPSRC under grant EP/G042705/1 'Increased Reliability for Industrially Relevant Automatic Crack Growth Simulation with the eXtended Finite Element Method' and the European Research Council Starting Independent Research Grant (ERC Stg grant agreement No. 279578) entitled 'Towards real time multiscale 
simulation of cutting in non-linear materials with applications to surgical simulation and computer guided surgery'. E. Atroshchenko was partially supported by Fondecyt grant number 11130259 entitled 'Boundary element modeling of crack propagation in micropolar materials'.

\section{Appendix A. Auxiliary displacement and stress fields in $M$ integral}

The auxiliary stress field $\sigma_{i j}^{(2)}$ and displacement field $u_{j}^{(2)}$ are given as:

$$
\begin{aligned}
\sigma_{x x} & =\frac{K_{I}^{(2)}}{\sqrt{2 \pi r}} \cos \frac{\theta}{2}\left(1-\sin \frac{\theta}{2} \sin \frac{3 \theta}{2}\right)-\frac{K_{I I}^{(2)}}{\sqrt{2 \pi r}} \sin \frac{\theta}{2}\left(2+\cos \frac{\theta}{2} \cos \frac{3 \theta}{2}\right) \\
\sigma_{y y} & =\frac{K_{I}^{(2)}}{\sqrt{2 \pi r}} \cos \frac{\theta}{2}\left(1+\sin \frac{\theta}{2} \sin \frac{3 \theta}{2}\right)+\frac{K_{I I}^{(2)}}{\sqrt{2 \pi r}} \sin \frac{\theta}{2} \cos \frac{\theta}{2} \cos \frac{3 \theta}{2}, \\
\tau_{x y} & =\frac{K_{I}^{(2)}}{\sqrt{2 \pi r}} \sin \frac{\theta}{2} \cos \frac{\theta}{2} \cos \frac{3 \theta}{2}+\frac{K_{I I}^{(2)}}{\sqrt{2 \pi r}} \cos \frac{\theta}{2}\left(1-\sin \frac{\theta}{2} \sin \frac{3 \theta}{2}\right), \\
\tau_{y z} & =\frac{K_{I I I}^{(2)}}{\sqrt{2 \pi r}} \cos \frac{\theta}{2}, \\
\tau_{z x} & =-\frac{K_{I I I}^{(2)}}{\sqrt{2 \pi r}} \sin \frac{\theta}{2}, \\
\tau_{z z}= & v\left(\sigma_{x x}+\sigma_{y y}\right), \\
u_{x}= & \frac{K_{I}}{2 \mu} \sqrt{\frac{r}{2 \pi}} \cos \frac{\theta}{2}\left(\kappa-1+2 \sin ^{2} \frac{\theta}{2}\right)+\frac{(1+v) K_{I I}}{E} \sqrt{\frac{r}{2 \pi}} \sin \frac{\theta}{2}\left(\kappa+1+2 \cos ^{2} \frac{\theta}{2}\right), \\
u_{y}= & \frac{K_{I}}{2 \mu} \sqrt{\frac{r}{2 \pi}} \sin \frac{\theta}{2}\left(\kappa+1-2 \cos ^{2} \frac{\theta}{2}\right)+\frac{(1+v) K_{I I}}{E} \sqrt{\frac{r}{2 \pi}} \cos \frac{\theta}{2}\left(1-\kappa+2 \sin ^{2} \frac{\theta}{2}\right), \\
u_{z}= & \frac{2 K_{I I I}}{\mu} \sqrt{\frac{r}{2 \pi}} \sin \frac{\theta}{2}
\end{aligned}
$$

where $(r, \theta)$ are the crack tip polar coordinates and $\mu=\frac{E}{2(1+v)}, \kappa=3-4 v$.

The auxiliary strain field can be obtained by differentiating $u_{j}$ with respect to the physical coordinate.

\section{Appendix B. Geometry data}

See Tables 3 and 4.

Table 3

Control points for NURBS-represented penny crack.

The knot vectors are: angular direction $\xi=$ $[0,0,0,0.25,0.25,0.5,0.5,0.75,0.75,1,1,1]$, radial direction $\boldsymbol{\eta}=[0,0,0,1,1,1]$.

\begin{tabular}{llll}
\hline$i$ & $j$ & $P_{i, j}$ & $w_{i, j}$ \\
\hline 0 & 0 & $(0.0,0.0)$ & 1.0 \\
1 & 0 & $(0.0,0.0)$ & 0.707106 \\
2 & 0 & $(0.0,0.0)$ & 1.0 \\
3 & 0 & $(0.0,0.0)$ & 0.707106 \\
4 & 0 & $(0.0,0.0)$ & 1.0 \\
5 & 0 & $(0.0,0.0)$ & 0.707106 \\
6 & 0 & $(0.0,0.0)$ & 1.0 \\
7 & 0 & $(0.0,0.0)$ & 0.707106
\end{tabular}

(continued on next page) 
Table 3 (continued)

\begin{tabular}{llll}
\hline$i$ & $j$ & $P_{i, j}$ & $w_{i, j}$ \\
\hline 8 & 0 & $(0.0,0.0)$ & 1.0 \\
0 & 1 & $(0.025,0.0)$ & 1.0 \\
1 & 1 & $(0.025,-0.025)$ & 0.707106 \\
2 & 1 & $(0,-0.025)$ & 1.0 \\
3 & 1 & $(-0.025,-0.025)$ & 0.707106 \\
4 & 1 & $(-0.025,0.0)$ & 1.0 \\
5 & 1 & $(-0.025,0.025)$ & 0.707106 \\
6 & 1 & $(0.0,0.025,0.0)$ & 1.0 \\
7 & 1 & $(0.025,0.025)$ & 0.707106 \\
8 & 1 & $(0.025,0.0)$ & 1.0 \\
0 & 2 & $(0.05,0.0)$ & 1.0 \\
1 & 2 & $(0.05,-0.05)$ & 0.707106 \\
2 & 2 & $(0.0,-0.05)$ & 1.0 \\
3 & 2 & $(-0.05,-0.05)$ & 0.707106 \\
4 & 2 & $(-0.05,0.0)$ & 1.0 \\
5 & 2 & $(-0.05,0.05)$ & 0.707106 \\
6 & 2 & $(0.0,0.05)$ & 1.0 \\
7 & 2 & $(0.05,0.05)$ & 0.707106 \\
8 & 2 & $(0.05,0.0)$ & 1.0 \\
\hline
\end{tabular}

Table 4

Control points for NURBS-represented elliptical crack. The knot vectors are: angular direction $\boldsymbol{\xi}=$ $[0,0,0,0.25,0.25,0.5,0.5,0.75,0.75,1,1,1]$, radial direction $\eta=[0,0,0,1,1,1]$.

\begin{tabular}{llll}
\hline$i$ & $j$ & $P_{i, j}$ & $w_{i, j}$ \\
\hline 0 & 0 & $(0.0,0.0)$ & 1.0 \\
1 & 0 & $(0.0,0.0)$ & 0.707106 \\
2 & 0 & $(0.0,0.0)$ & 1.0 \\
3 & 0 & $(0.0,0.0)$ & 0.707106 \\
4 & 0 & $(0.0,0.0)$ & 1.0 \\
5 & 0 & $(0.0,0.0)$ & 0.707106 \\
6 & 0 & $(0.0,0.0)$ & 1.0 \\
7 & 0 & $(0.0,0.0)$ & 0.707106 \\
8 & 0 & $(0.0,0.0)$ & 1.0 \\
0 & 1 & $(0.025,0.0)$ & 1.0 \\
1 & 1 & $(0.025,-0.0125)$ & 0.707106 \\
2 & 1 & $(0,-0.0125)$ & 1.0 \\
3 & 1 & $(-0.025,-0.0125)$ & 0.707106 \\
4 & 1 & $(-0.025,0.0)$ & 1.0 \\
5 & 1 & $(-0.025,0.0125)$ & 0.707106 \\
6 & 1 & $(0.0,0.0125,0.0)$ & 1.0 \\
7 & 1 & $(0.025,0.0125)$ & 0.707106 \\
8 & 1 & $(0.025,0.0)$ & 1.0 \\
0 & 2 & $(0.05,0.0)$ & 1.0 \\
1 & 2 & $(0.05,-0.025)$ & 0.707106 \\
2 & 2 & $(0.0,-0.025)$ & 1.0 \\
3 & 2 & $(-0.05,-0.025)$ & 0.707106 \\
4 & 2 & $(-0.05,0.0)$ & 1.0 \\
5 & 2 & $(-0.05,0.025)$ & 0.707106 \\
6 & 2 & $(0.0,0.025)$ & 1.0 \\
7 & 2 & $(0.05,0.025)$ & 0.707106 \\
8 & 2 & $(0.05,0.0)$ & 1.0 \\
\hline & & &
\end{tabular}




\section{References}

[1] T. Rabczuk, S. Bordas, G. Zi, On three-dimensional modelling of crack growth using partition of unity methods, Comput. Struct. 88 (23-24) (2010) 1391-1411.

[2] Roshdy S. Barsoum, Further application of quadratic isoparametric finite elements to linear fracture mechanics of plate bending and general shells, Int. J. Fract. 11 (1) (1975) 167-169.

[3] L.F. Martha, P.A. Wawrzynek, A.R. Ingraffea, Arbitrary crack representation using solid modeling, Eng. Comput. 9 (2) (1993) 63-82.

[4] V. Chiaruttini, V. Riolo, F. Feyel, Advanced remeshing techniques for complex 3D crack propagation, in: ICF13, 2013.

[5] A.R. Khoei, M. Eghbalian, H. Moslemi, H. Azadi, Crack growth modeling via 3D automatic adaptive mesh refinement based on modifiedSPR technique, Appl. Math. Model. 37 (1) (2013) 357-383.

[6] M. Schöllmann, M. Fulland, H.A. Richard, Development of a new software for adaptive crack growth simulations in 3D structures, Eng Fract. Mech. 70 (2) (2003) 249-268.

[7] A.R. Maligno, S. Rajaratnam, S.B. Leen, E.J. Williams, A three-dimensional (3D) numerical study of fatigue crack growth using remeshing techniques, Eng. Fract. Mech. 77 (1) (2010) 94-111.

[8] B.J. Carter, P.A. Wawrzynek, A.R. Ingraffea, Automated 3-D crack growth simulation, Internat. J. Numer. Methods Engrg. 47 (1-3) (2000) 229-253.

[9] R. Branco, F.V. Antunes, J.D. Costa, A review on 3D-FE adaptive remeshing techniques for crack growth modelling, Eng. Fract. Mech. 141 (2015) 170-195.

[10] J.F. Remacle, V. Bertrand, C. Geuzaine, A two-level multithreaded delaunay kernel, Procedia Eng. 124 (2015) 6-17.

[11] J. Pellerin, B. Lévy, G. Caumon, A. Botella, Automatic surface remeshing of 3D structural models at specified resolution: A method based on Voronoi diagrams, Comput. Geosci. 62 (2014) 103-116.

[12] J.M. Melenk, I. Babuška, The partition of unity finite element method: Basic theory and applications, Comput. Methods Appl. Mech. Engrg 139 (1-4) (1996) 289-314.

[13] N. Moës, J. Dolbow, T. Belytschko, A finite element method for crack growth without remeshing, Internat. J. Numer. Methods Engrg. 46 (1) (1999) 131-150.

[14] C.A. Duarte, I. Babuška, J.T. Oden, Generalized finite element methods for three-dimensional structural mechanics problems, Computers \& Structures 77 (2) (2000) 215-232.

[15] T. Strouboulis, K. Copps, I. Babuška, The generalized finite element method, Comput. Methods Appl. Mech. Engrg. 190 (32-33) (2001) 4081-4193.

[16] G. Strang, G. Fix, An Analysis of the Finite Element Method, second ed., Wellesley-Cambridge, 2008.

[17] M. Fleming, Y.A. Chu, B. Moran, T. Belytschko, enriched element-free Galerkin methods for crack tip fields, Internat. J. Numer. Methods Engrg. 40 (8) (1997) 1483-1504.

[18] V.P. Nguyen, T. Rabczuk, S. Bordas, M. Duflot, Meshless methods: A review and computer implementation aspects, Math. Comput. Simulation 79 (3) (2008) 763-813.

[19] J.J. Ródenas, O.A. González-Estrada, J.E. Tarancón, F.J. Fuenmayor, A recovery-type error estimator for the extended finite element method based on singular + smooth stress field splitting, Internat. J. Numer. Methods Engrg. 76 (4) (2008) 545-571.

[20] M. Duflot, S.P.A. Bordas, A posteriori error estimation for extended finite elements by an extended global recovery, Internat. J. Numer. Methods Engrg. 76 (8) (2008) 1123-1138.

[21] S.P.A. Bordas, M. Duflot, Derivative recovery and a posteriori error estimate for extended finite elements, Comput. Methods Appl. Mech. Engrg. 196 (35-36) (2007) 3381-3399.

[22] S. Natarajan, S.P.A. Bordas, D.R. Mahapatra, Numerical integration over arbitrary polygonal domains based on Schwarz-Christoffel conformal mapping, Internat. J. Numer. Methods Engrg. 80 (1) (2009) 103-134.

[23] G. Ventura, R. Gracie, T. Belytschko, Fast integration and weight function blending in the extended finite element method, Internat. J. Numer. Methods Engrg. 77 (1) (2009) 1-29.

[24] R. Gracie, H. Wang, T. Belytschko, Blending in the extended finite element method by discontinuous Galerkin and assumed strain methods, Internat. J. Numer. Methods Engrg. 74 (11) (2008) 1645-1669.

[25] A. Menk, S.P.A. Bordas, A robust preconditioning technique for the extended finite element method, Internat. J. Numer. Methods Engrg. 85 (13) (2011) 1609-1632.

[26] V. Gupta, C.A. Duarte, I. Babuška, U. Banerjee, Stable GFEM (SGFEM): Improved conditioning and accuracy of GFEM/XFEM for threedimensional fracture mechanics, Comput. Methods Appl. Mech. Engrg. 289 (2015) 355-386.

[27] K. Agathos, E. Chatzi, S.P.A. Bordas, D. Talaslidis, A well-conditioned and optimally convergent XFEM for 3D linear elastic fracture, Internat. J. Numer. Methods Engrg. 105 (9) (2015) 643-677.

[28] N. Sukumar, N. Moës, B. Moran, T. Belytschko, Extended finite element method for three-dimensional crack modelling, Internat. J. Numer. Methods Engrg. 48 (11) (2000) 1549-1570.

[29] N. Sukumar, D.L. Chopp, E. Béchet, N. Moës, Three-dimensional non-planar crack growth by a coupled extended finite element and fast marching method, Internat. J. Numer. Methods Engrg. 76 (5) (2008) 727-748.

[30] A. Gravouil, N. Moës, T. Belytschko, Non-planar 3D crack growth by the extended finite element and level sets-Part II: Level set update, Internat. J. Numer. Methods Engrg. 53 (11) (2002) 2569-2586.

[31] N. Moës, A. Gravouil, T. Belytschko, Non-planar 3D crack growth by the extended finite element and level sets-Part I: Mechanical model, Internat. J. Numer. Methods Engrg. 53 (11) (2002) 2549-2568

[32] S. Bordas, B. Moran, Enriched finite elements and level sets for damage tolerance assessment of complex structures, Eng. Fract. Mech. 73 (9) (2006) 1176-1201.

[33] E. Wyart, M. Duflot, D. Coulon, P. Martiny, T. Pardoen, J.-F. Remacle, F. Lani, Substructuring FE-XFE approaches applied to threedimensional crack propagation, J. Comput. Appl. Math. 215 (2) (2008) 626-638. 
[34] P. Krysl, T. Belytschko, The element free Galerkin method for dynamic propagation of arbitrary 3-D cracks, Internat. J. Numer. Methods Engrg. 44 (6) (1999) 767-800.

[35] G. Ventura, J.X. Xu, T. Belytschko, A vector level set method and new discontinuity approximations for crack growth by EFG, Internat. J. Numer. Methods Engrg. 54 (6) (2002) 923-944.

[36] S.P.A. Bordas, T. Rabczuk, G. Zi, Three-dimensional crack initiation, propagation, branching and junction in non-linear materials by an extended meshfree method without asymptotic enrichment, Eng. Fract. Mech. 75 (5) (2008) 943-960.

[37] T. Rabczuk, G. Zi, S. Bordas, H. Nguyen-Xuan, A simple and robust three-dimensional cracking-particle method without enrichment, Comput. Methods Appl. Mech. Engrg. 199 (37-40) (2010) 2437-2455.

[38] H. Hong, J. Chen, Derivations of integral equations of elasticity, J. Eng. Mech. 114 (6) (1988) 1028-1044.

[39] J.T. Chen, H.K. Hong, Review of dual boundary element methods with emphasis on hypersingular integrals and divergent series, Appl. Mech. Rev. 52 (1) (1999) 17-33.

[40] T.J. Rudolphi, The use of simple solutions in the regularization of hypersingular boundary integral equations, Math. Comput. Modelling 15 (3-5) (1991) 269-278.

[41] Y. Liu, T.J. Rudolphi, Some identities for fundamental solutions and their applications to weakly-singular boundary element formulations, Eng. Anal. Bound. Elem. 8 (6) (1991) 301-311.

[42] B. Marussig, J. Zechner, G. Beer, T.P. Fries, Fast isogeometric boundary element method based on independent field approximation, Comput. Methods Appl. Mech. Engrg. 284 (2015) 458-488.

[43] A. Portela, M.H. Aliabadi, D.P. Rooke, The dual boundary element method: Effective implementation for crack problems, Internat. J. Numer. Methods Engrg. 33 (6) (1992) 1269-1287.

[44] Y. Mi, M.H. Aliabadi, Dual boundary element method for three-dimensional fracture mechanics analysis, Engineering Analysis with Boundary Elements 10 (2) (1992) 161-171.

[45] A.P. Cisilino, M.H. Aliabadi, Dual boundary element assessment of three-dimensional fatigue crack growth, Engineering Analysis with Boundary Elements 28 (9) (2004) 1157-1173.

[46] A.P. Cisilino, M.H. Aliabadi, Three-dimensional boundary element analysis of fatigue crack growth in linear and non-linear fracture problems, Eng. Fract. Mech. 63 (6) (1999) 713-733.

[47] V. Leitão, M.H. Aliabadi, D.P. Rooke, The dual boundary element formulation for elastoplastic fracture mechanics, Internat. J. Numer. Methods Engrg. 38 (2) (1995) 315-333.

[48] P. Fedelinski, M.H. Aliabadi, D.P. Rooke, The dual boundary element method in dynamic fracture mechanics, Engineering Analysis with Boundary Elements 12 (3) (1993) 203-210.

[49] S.C. Mellings, J.M.W. Baynham, Automatic fatigue crack growth, in: ASME 2009 Pressure Vessels and Piping Conference, American Society of Mechanical Engineers, 2009, pp. 1513-1523.

[50] S. Li, M.E. Mear, L. Xiao, Symmetric weak-form integral equation method for three-dimensional fracture analysis, Comput. Methods Appl. Mech. Engrg. 151 (3-4) (1998) 435-459.

[51] A. Frangi, Fracture propagation in 3D by the symmetric Galerkin boundary element method, International Journal of Fracture 116 (4) (2002) $313-330$

[52] A. Frangi, G. Novati, R. Springhetti, M. Rovizzi, 3D fracture analysis by the symmetric Galerkin BEM, Comput. Mech. 28 (3-4) (2002) $220-232$

[53] G.P. Nikishkov, J.H. Park, S.N. Atluri, SGBEM-FEM alternating method for analyzing 3D non-planar cracks and their growth in structural components, Comput. Model. Eng. Sci. 2 (3) (2001) 401-422.

[54] A. Frangi, G. Novati, BEM-FEM coupling for 3D fracture mechanics applications, Comput. Mech. 32 (4-6) (2003) 415-422.

[55] C. Song, J.P. Wolf, The scaled boundary finite-element method-alias consistent infinitesimal finite-element cell method-for elastodynamics, Comput. Methods Appl. Mech. Engrg. 147 (3-4) (1997) 329-355.

[56] S. Natarajan, J. Wang, C. Song, C. Birk, Isogeometric analysis enhanced by the scaled boundary finite element method, Comput. Methods Appl. Mech. Engrg. 283 (2015) 733-762.

[57] T.J.R. Hughes, J.A. Cottrell, Y. Bazilevs, Isogeometric analysis: CAD, finite elements, NURBS, exact geometry and mesh refinement, Comput. Methods Appl. Mech. Engrg. 194 (39-41) (2005) 4135-4195.

[58] E. De Luycker, D.J. Benson, T. Belytschko, Y. Bazilevs, M.C. Hsu, X-FEM in isogeometric analysis for linear fracture mechanics, Internat. J. Numer. Methods Engrg. 87 (6) (2011) 541-565.

[59] S. Ghorashi, N. Valizadeh, S. Mohammadi, Extended isogeometric analysis for simulation of stationary and propagating cracks, Internat. J. Numer. Methods Engrg. 89 (9) (2012) 1069-1101.

[60] V.P. Nguyen, C. Anitescu, S.P.A. Bordas, T. Rabczuk, Isogeometric analysis: An overview and computer implementation aspects, Math. Comput. Simulation 117 (2015) 89-116.

[61] N. Nguyen-Thanh, N. Valizadeh, M.N. Nguyen, H. Nguyen-Xuan, X. Zhuang, P. Areias, G. Zi, Y. Bazilevs, L. De Lorenzis, T. Rabczuk, An extended isogeometric thin shell analysis based on Kirchhoff-Love theory, Comput. Methods Appl. Mech. Engrg. 284 (2015) $265-291$.

[62] R.N. Simpson, S.P.A. Bordas, J. Trevelyan, T. Rabczuk, A two-dimensional isogeometric boundary element method for elastostatic analysis, Comput. Methods Appl. Mech. Engrg. 209-212 (0) (2012) 87-100.

[63] H. Lian, R.N. Simpson, S.P.A. Bordas, Stress analysis without meshing: isogeometric boundary-element method, Proc. Inst. Civil Eng. Engrg. Comput. Mech. 166 (2) (2013) 88-99.

[64] M.A. Scott, R.N. Simpson, J.A. Evans, S. Lipton, S.P.A. Bordas, T.J.R. Hughes, T.W. Sederberg, Isogeometric boundary element analysis using unstructured T-splines, Comput. Methods Appl. Mech. Engrg. 254 (0) (2013) 197-221.

[65] K. Li, X. Qian, Isogeometric analysis and shape optimization via boundary integral, Comput.-Aided Des. 43 (11) (2011) $1427-1437$.

[66] K.V. Kostas, A.I. Ginnis, C.G. Politis, P.D. Kaklis, Ship-hull shape optimization with a T-spline based BEM-isogeometric solver, Comput. Methods Appl. Mech. Engrg. 284 (2015) 611-622. 
[67] H. Lian, P. Kerfriden, S.P.A. Bordas, Implementation of regularized isogeometric boundary element methods for gradient-based shape optimization in two-dimensional linear elasticity, Internat. J. Numer. Methods Engrg. 106 (12) (2016) 972-1017.

[68] R.N. Simpson, M.A. Scott, M. Taus, D.C. Thomas, H. Lian, Acoustic isogeometric boundary element analysis, Comput. Methods Appl. Mech. Engrg. (2013) page accepted.

[69] J. Gu, J. Zhang, G. Li, Isogeometric analysis in BIE for 3-D potential problem, Engineering Analysis with Boundary Elements 36 (5) (2012) 858-865.

[70] C. Politis, A.I. Ginnis, P.D. Kaklis, K. Belibassakis, C. Feurer, An isogeometric BEM for exterior potential-flow problems in the plane, in: 2009 SIAM/ACM Joint Conference on Geometric and Physical Modeling, SPM'09, ACM, New York, NY, USA, 2009 , pp. $349-354$.

[71] A.I. Ginnis, K.V. Kostas, C.G. Politis, P.D. Kaklis, K.A. Belibassakis, Th.P. Gerostathis, M.A. Scott, T.J.R. Hughes, Isogeometric boundaryelement analysis for the wave-resistance problem using T-splines, Comput. Methods Appl. Mech. Engrg. 279 (2014) $425-439$.

[72] M.J. Peake, J. Trevelyan, G. Coates, Extended isogeometric boundary element method (XIBEM) for two-dimensional Helmholtz problems, Comput. Methods Appl. Mech. Engrg. 259 (0) (2013) 93-102.

[73] M.J. Peake, J. Trevelyan, G. Coates, Extended isogeometric boundary element method (XIBEM) for three-dimensional medium-wave acoustic scattering problems, Comput. Methods Appl. Mech. Engrg. 284 (2015) 762-780.

[74] G. Beer, B. Marussig, J. Zechner, A simple approach to the numerical simulation with trimmed CAD surfaces, Comput. Methods Appl. Mech. Engrg. 285 (2015) 776-790.

[75] Y. Wang, D.J. Benson, A.P. Nagy, A multi-patch nonsingular isogeometric boundary element method using trimmed elements, Comput. Mech. 56 (1) (2015) 173-191.

[76] M. Feischl, G. Gantner, D. Praetorius, Reliable and efficient a posteriori error estimation for adaptive $\{$ IGA $\}$ boundary element methods for weakly-singular integral equations, Comput. Methods Appl. Mech. Engrg. 290 (2015) 362-386.

[77] A. Aimi, M. Diligenti, M.L. Sampoli, A. Sestini, Isogemetric analysis and symmetric Galerkin BEM: A 2D numerical study, Appl. Math. Comput. (2015).

[78] M. Taus, G.J. Rodin, T.J.R. Hughes, Isogeometric Analysis of Boundary Integral Equations, ICES Report 15-12, 2015.

[79] B.H. Nguyen, H.D. Tran, C. Anitescu, X. Zhuang, T. Rabczuk, An isogeometric symmetric Galerkin boundary element method for twodimensional crack problems, Comput. Methods Appl. Mech. Engrg. 306 (2016) 252-275.

[80] L. Liu, Y. Zhang, T.J.R. Hughes, M.A. Scott, T.W. Sederberg, Volumetric T-spline construction using Boolean operations, Eng. Comput. 30 (4) (2013) 425-439.

[81] G. Xu, B. Mourrain, R. Duvigneau, A. Galligo, Analysis-suitable volume parameterization of multi-block computational domain in isogeometric applications, Comput.-Aided Des. 45 (2) (2013) 395-404.

[82] G. Xu, B. Mourrain, R. Duvigneau, A. Galligo, Constructing analysis-suitable parameterization of computational domain from CAD boundary by variational harmonic method, J. Comput. Phys. 252 (2013) 275-289.

[83] M. Guiggiani, Formulation and numerical treatment of boundary integral equations with hypersingular kernels, in: Singular Integrals in Boundary Element Methods, 1998, pp. 85-124.

[84] L. Piegl, W. Tiller, The NURBS Book, Springer, 1995.

[85] Y.J. Wang, D.J. Benson, Multi-patch nonsingular isogeometric boundary element analysis in 3D, Comput. Methods Appl. Mech. Engrg. 293 (2015) 71-91.

[86] M. Guiggiani, G. Krishnasamy, T.J. Rudolphi, F.J. Rizzo, A general algorithm for the numerical solution of hypersingular boundary integral equations, J. Appl. Mech. 59 (3) (1992) 604-614.

[87] J. Rong, L. Wen, J. Xiao, Efficiency improvement of the polar coordinate transformation for evaluating BEM singular integrals on curved elements, Engineering Analysis With Boundary Elements 38 (2014) 83-93.

[88] S. Osher, R. Fedkiw, Level Set Methods and Dynamic Implicit Surfaces, Vol. 153, Springer Science \& Business Media, 2006.

[89] N. Moës, M. Cloirec, P. Cartraud, J.F. Remacle, A computational approach to handle complex microstructure geometries, Comput. Methods Appl. Mech. Engrg. 192 (28-30) (2003) 3163-3177.

[90] T.J. Barth, J.A. Sethian, Numerical schemes for the Hamilton-Jacobi and level set equations on triangulated domains, J. Comput. Phys. 145 (1) (1998) 1-40.

[91] D.L. Chopp, N. Sukumar, Fatigue crack propagation of multiple coplanar cracks with the coupled extended finite element/fast marching method, Internat. J. Engrg. Sci. 41 (8) (2003) 845-869.

[92] T.P. Fries, M. Baydoun, Crack propagation with the extended finite element method and a hybrid explicit-implicit crack description, Internat. J. Numer. Methods Engrg. 89 (12) (2012) 1527-1558.

[93] A. Paluszny, R.W. Zimmerman, Numerical simulation of multiple 3D fracture propagation using arbitrary meshes, Comput. Methods Appl. Mech. Engrg. 200 (9-12) (2011) 953-966.

[94] J. Garzon, P. O’Hara, C.A. Duarte, W.G. Buttlar, Improvements of explicit crack surface representation and update within the generalized finite element method with application to three-dimensional crack coalescence, Internat. J. Numer. Methods Engrg. 97 (4) (2014) $231-273$.

[95] A. Paluszny, R.W. Zimmerman, Numerical fracture growth modeling using smooth surface geometric deformation, Eng. Fract. Mech. 108 (0) (2013) 19-36.

[96] R. LaGreca, M. Daniel, A. Bac, Local deformation of NURBS curves, in: Mathematical Methods for Curves and Surfaces, Tromso 2004, 2005 , pp. 243-252.

[97] A. Tambat, G. Subbarayan, Isogeometric enriched field approximations, Comput. Methods Appl. Mech. Engrg. 245-246 (2012) 1-21.

[98] K. Upreti, T. Song, A. Tambat, G. Subbarayan, Algebraic distance estimations for enriched isogeometric analysis, Comput. Methods Appl. Mech. Engrg. 280 (2014) 28-56.

[99] S.K. Chan, I.S. Tuba, W.K. Wilson, On the finite element method in linear fracture mechanics, Eng. Fract. Mech. 2 (1) (1970) $1-17$.

[100] T.K. Hellen, On the method of virtual crack extensions, Internat. J. Numer. Methods Engrg 9 (1) (1975) $187-207$.

[101] C.G. Hwang, P.A. Wawrzynek, A.R. Ingraffea, On the virtual crack extension method for calculating the derivatives of energy release rates for a 3D planar crack of arbitrary shape under mode-I loading, Eng. Fract. Mech. 68 (7) (2001) 925-947. 
[102] B.R. Davis, P.A. Wawrzynek, A.R. Ingraffea, 3-D simulation of arbitrary crack growth using an energy-based formulation - Part I: Planar growth, Eng. Fract. Mech. 115 (2014) 204-220.

[103] B.R. Davis, P.A. Wawrzynek, A.R. Ingraffea, Simulation of arbitrary mixed-mode crack growth using an energy-based approach, in: Jay Carroll, Samantha Daly (Eds.), Fracture, Fatigue, Failure, and Damage Evolution, Vol. 5, in: Conference Proceedings of the Society for Experimental Mechanics Series, Springer International Publishing, 2015, pp. 1-9.

[104] D. Sutula, S.P.A. Bordas, P. Kerfriden, A. Barthelemy, Global energy minimization for multi-crack growth in linear elastic fracture using the extended finite element methods, in: 11th World Congress on Computational Mechanics (WCCM XI), Barcelona, 2014.

[105] R. Krueger, Virtual crack closure technique: history, approach, and applications, Appl. Mech. Rev. 57 (2) (2004) 109-143.

[106] J.R. Rice, A path independent integral and the approximate analysis of strain concentration by notches and cracks, J. Appl. Mech. 35 (2) (1968) 379-386.

[107] M. Stern, E.B. Becker, R.S. Dunham, A contour integral computation of mixed-mode stress intensity factors, International Journal of Fracture 12 (3) (1976) 359-368.

[108] J.F. Yau, S.S. Wang, H.T. Corten, A mixed-mode crack analysis of isotropic solids using conservation laws of elasticity, J. Appl. Mech. 47 (1980) 333-341.

[109] P.H. Wen, M.H. Aliabadi, D.P. Rooke, A contour integral for the evaluation of stress intensity factors, Appl. Math. Model. 19 (8) (1995) 450-455.

[110] I. Babuška, A. Miller, The post-processing approach in the finite element method. Part 2: The calculation of stress intensity factors, International Journal for numerical methods in Engineering 20 (6) (1984) 1111-1129.

[111] J.P. Pereira, C.A. Duarte, Extraction of stress intensity factors from generalized finite element solutions, Engineering Analysis with Boundary Elements 29 (4) (2005) 397-413.

[112] R.H. Rigby, M.H. Aliabadi, Mixed-mode J-integral method for analysis of 3D fracture problems using BEM, Engineering Analysis with Boundary Elements 11 (3) (1993) 239-256.

[113] J.H. Chang, D.J. Wu, Stress intensity factor computation along a non-planar curved crack in three dimensions, Int. J. Solids Struct. 44 (2) (2007) 371-386.

[114] G.P. Nikishkov, S.N. Atluri, Calculation of fracture mechanics parameters for an arbitrary three-dimensional crack, by the 'equivalent domain integral' method, Internat. J. Numer. Methods Engrg. 24 (9) (1987) 1801-1821.

[115] K.N. Shivakumar, I.S. Raju, An equivalent domain integral method for three-dimensional mixed-mode fracture problems, Eng. Fract. Mech. 42 (6) (1992) 935-959.

[116] O. Huber, J. Nickel, G. Kuhn, On the decomposition of the J-integral for 3D crack problems, International Journal of Fracture 64 (4) (1993) 339-348.

[117] R.H. Rigby, M.H. Aliabadi, Decomposition of the mixed-mode J-integral—revisited, Int. J. Solids Struct. 35 (17) (1998) $2073-2099$

[118] M. Gosz, B. Moran, An interaction energy integral method for computation of mixed-mode stress intensity factors along non-planar crack fronts in three dimensions, Eng. Fract. Mech. 69 (3) (2002) 299-319.

[119] A.P. Cisilino, J. Ortiz, Boundary element analysis of three-dimensional mixed-mode cracks via the interaction integral, Comput. Methods Appl. Mech. Engrg. 194 (9-11) (2005) 935-956.

[120] L.A. de Lacerda, L.C. Wrobel, Dual boundary element method for axisymmetric crack analysis, International Journal of Fracture 113 (3) (2002) 267-284

[121] F. Erdogan, G. Sih, On the crackextension in plates under plane loading and transverse shear, J. Basic Eng. 85 (1963) $519-527$.

[122] T.N. Farris, M. Liu, Boundary element crack closure calculation of three-dimensional stress intensity factors, International journal of fracture 60 (1) (1993) 33-47.

[123] N. Sukumar, D.L. Chopp, B. Moran, Extended finite element method and fast marching method for three-dimensional fatigue crack propagation, Eng. Fract. Mech. 70 (1) (2003) 29-48. 